\title{
Optical - near-infrared catalog for the AKARI north ecliptic pole Deep field ${ }^{\star}$
}

\author{
Nagisa $\mathrm{Oi}^{1}$, Hideo Matsuhara ${ }^{1}$, Kazumi Murata ${ }^{1,2}$, Tomotsugu Goto ${ }^{3}$, Takehiko Wada ${ }^{1}$, Toshinobu Takagi ${ }^{1}$, \\ Youichi Ohyama ${ }^{4}$, Matthew Malkan ${ }^{5}$, Myungshin $\operatorname{Im}^{6}$, Hyunjin Shim ${ }^{6,7}$, Stephen Serjeant ${ }^{8}$, and Chris Pearson ${ }^{8,9,10}$ \\ ${ }^{1}$ Institute of Space and Astronautical Science, Japan Aerospace Exploration Agency, Sagamihara, 252-5210 Kanagawa, Japan \\ e-mail: nagisaoi@ir.isas.jaxa.jp \\ 2 Department of Space and Astronautical Science, The Graduate University for Advanced Studies, 753-8511 Yamaguchi, Japan \\ 3 Institute of Astronomy and Department of Physics, National Tsing Hua University No. 101, Section 2, Kuang-Fu Road, \\ Hsinchu 30013, Taiwan, R.O.C. \\ 4 Academia Sinica, Institute of Astronomy and Astrophysics, 11F of Astronomy-Mathematics Building, National Taiwan University, \\ No.1, Sec. 4, Roosevelt Rd, 10617 Taipei, Taiwan R.O.C. \\ 5 Department of Physics and Astronomy, UCLA, Los Angeles, CA 90095-1547, USA \\ 6 Department of Physics \& Astronomy, FPRD, Seoul National University, Shillim-Dong, Kwanak-Gu, 151-742 Seoul, Korea \\ 7 Department of Earth Science Education, Kyungpook National University, 702-701 Daegu, Republic of Korea \\ 8 Astrophysics Group, Department of Physics, The Open University, Milton Keynes, MK7 6AA, UK \\ 9 RAL Space, Rutherford Appleton Laboratory, Harwell Oxford, OX11 0QZ, UK \\ 10 Oxford Astrophysics, Oxford University, Keble Road, Oxford OX1 3RH, UK \\ Received 29 August 2013 / Accepted 18 March 2014
}

\section{ABSTRACT}

\begin{abstract}
Aims. We present an 8-band $\left(u^{*}, g^{\prime}, r^{\prime}, i^{\prime}, z^{\prime}, Y, J, K_{\mathrm{s}}\right)$ optical to near-infrared deep photometric catalog based on the observations made with MegaCam and WIRCam at the CFHT, and compute photometric redshifts, $z_{\mathrm{p}}$ in the north ecliptic pole (NEP) region. AKARI infrared satellite carried out a deep survey in the NEP region at near- to mid-infrared wavelengths. Our optical to nearinfrared catalog allows us to identify the counterparts and $z_{\mathrm{p}}$ for the AKARI sources.

Methods. We obtained seven-band $\left(g^{\prime}, r^{\prime}, i^{\prime}, z^{\prime}, Y, J, K_{\mathrm{S}}\right)$ imaging data, and we crossmatched them with existing $u^{*}$-band data (limiting magnitude $=24.6 \mathrm{mag}[5 \sigma ; \mathrm{AB}])$ to design the band-merged catalog. We included all $z^{\prime}$-band sources with counterparts in at least one of the other bands in the catalog. We used a template-fitting methods to compute $z_{\mathrm{p}}$ for all the cataloged sources.

Results. The estimated $4 \sigma$ detection limits within a 1 arcsec aperture radius are 26.7, 25.9, 25.1, and $24.1 \mathrm{mag}$ [AB] for the optical $g^{\prime}, r^{\prime}, i^{\prime}$, and $z^{\prime}$-bands and 23.4, 23.0, and $22.7 \mathrm{mag}$ for the near-infrared $Y, J$, and $K_{\mathrm{s}}$-bands, respectively. There are a total of 85797 sources in the band-merged catalog. An astrometric accuracy of this catalog determined by examining coordinate offsets with regard to 2MASS is 0.013 arcsec with a root mean square offset of 0.32 arcsec. We distinguish 5441 secure stars from extended sources using the $u^{*}-J$ versus $g^{\prime}-K_{\mathrm{s}}$ colours, combined with the SExtractor stellarity index of the images. Comparing with galaxy spectroscopic redshifts, we find a photometric redshift dispersion, $\sigma_{\Delta z /(1+z)}$, of 0.032 and catastrophic failure rate, $\frac{\Delta z}{1+z}>0.15$, of $5.8 \%$ at $z<1$, while a dispersion of 0.117 and a catastrophic failure rate of $16.6 \%$ at $z>1$. We extend the estimate of the $z_{\mathrm{p}}$ uncertainty over the full magnitude/redshift space with a redshift probability distribution function and find that our redshifts are highly accurate with $z^{\prime}<22$ at $z_{\mathrm{p}}<2.5$ and for fainter sources with $z^{\prime}<24$ at $z_{\mathrm{p}}<1$. From the investigation of photometric properties of AKARI infrared sources (23 354 sources) using the $g^{\prime} z^{\prime} K_{\mathrm{s}}$ diagram, $<5 \%$ of AKARI sources with optical counterparts are classified as high- $z$ $(1.4<z<2.5)$ star-forming galaxies. Among the high- $z$ star-forming galaxies, AKARI mid-infrared detected sources seem to be affected by stronger dust extinction compared with sources with non-detections in the AKARI mid-infrared bands.
\end{abstract}

Key words. methods: data analysis - catalogs - surveys - galaxies: photometry - infrared: galaxies

\section{Introduction}

An understanding of the cosmic history of star formation is one of the most important aspects in the study of galaxies. It has been shown that the star formation rate (SFR) density derived from ultraviolet (UV) and optical wavelengths at $z \sim 1$ is one order of magnitude higher than in the local Universe (Madau 1995; Hopkins et al. 2001; Connolly et al. 1997; Steidel et al. 1999; Ly et al. 2007). These results highlight the importance of studying the cosmic star formation activity at high redshift $(z \sim 1)$. Studies of the extragalactic background have suggested that at

$\star$ The full version of our 8-band merged catalog with $z_{\mathrm{p}}$ (Table 7) is available at the CDS via anonymous ftp to cdsarc.u-strasbg. fr $(130.79 .128 .5)$ or via

http://cdsarc.u-strasbg.fr/viz-bin/qcat?J/A+A/566/A60 least one third (or half) of the luminous energy generated by stars has been reprocessed into the infrared by dust (Lagache et al. 1999; Puget et al. 1996; Stecker et al. 2006; Franceschini et al. 2008) and that the contribution of infrared luminous sources to the cosmic infrared luminosity density increases with redshift, especially at $z>1$ (Goto et al. 2010). For luminous infrared galaxies $\left(L_{\mathrm{IR}} \gtrsim 10^{11} L_{\odot}\right)$, the SFR derived from UV continuum or optical diagnostics ( $\mathrm{H} \alpha$ emission line) is less than what is inferred from the infrared or radio luminosities, which are free from dust extinction. The tendency becomes more conspicuous with increasing infrared luminosity. This suggests that more luminous galaxies, i.e., galaxies with a higher SFR, are more heavily obscured by dust (Hopkins et al. 2001; Sullivan et al. 2001; Hicks et al. 2002; Choi et al. 2006; Buat et al. 2007). Therefore, to understand the star formation history, it is essential 
to investigate star formation in highly dust-obscured galaxies at high redshift $(z \gtrsim 1)$ in the mid-infrared (MIR) region where dust extinction is less severe.

The infrared space telescope AKARI (Murakami et al. 2007) was launched in February 2006 and the AKARI/IRC (InfraRed Camera: Onaka et al. 2007) obtained higher quality nearinfrared (NIR) to MIR data than the previous infrared space missions such as Infrared Astronomical Satellite (Neugebauer et al. 1984) and Infrared Space Observatory (Kessler et al. 1996). The IRC has three channels (NIR, MIR- $S$, and MIR- $L$ ) with nine filters $(N 2, N 3, N 4, S 7, S 9 W, S 11, L 15, L 18 W$, and $L 24)$, and AKARI carried out a deep survey program in the direction of north ecliptic pole (NEP), the so-called NEP-Deep survey (hereafter, NEP-Deep: Matsuhara et al. 2006) with all the nine IRC bands. Each single band observed $\sim 0.6$ square degrees, and the $\sim 0.5$ square degree circular area centered on $\alpha=17^{\mathrm{h}} 55^{\mathrm{m}} 24^{\mathrm{s}}$, $\delta=+66^{\circ} 37^{\prime} 32^{\prime \prime}$ are covered by all nine bands. The full width at half maximum (FWHM) of each IRC channel is $\sim 4$ arcsec in NIR, $\sim 5$ arcsec in MIR- $S$, and $\sim 6$ arcsec in MIR- $L$. Murata et al. (2013) constructed a revised NIR to MIR catalog of the AKARI NEP-Deep. The $5 \sigma$ detection limits in the AKARI NEP-Deep catalog are $13,10,12,34,38,64,98,105$, and $266 \mu \mathrm{Jy}$ at the $N 2, N 3, N 4, S 7, S 9 W, S 11, L 15, L 18 W$, and $L 24$ bands, respectively. The real strength of the NEP-Deep survey lies in its unprecedented photometric coverage from $2 \mu \mathrm{m}$ to $24 \mu \mathrm{m}$, which critically includes the wavelength domain between Spitzer IRAC (Fazio et al. 2004) and MIPS (Rieke et al. 2004) instruments from $8 \mu \mathrm{m}$ to $24 \mu \mathrm{m}$, where only limited coverage is available from the peak-up camera on the IRS (Houck et al. 2004). Therefore, NEP-Deep survey has the strong advantage of being able to study infrared galaxies without the uncertainties of interpolation over the MIR wavelength range.

Furthermore, the NEP-Deep field is a unique region because many astronomical satellites have accumulated many deep exposures that cover this location thanks to the nature of its position on the sky. X-ray observations by Chandra, UV observations by GALEX, and far-infrared observations by Herschel were made toward the NEP-Deep field. In addition, sub-mm data from SCUBA-2 on the James Clerk Maxwell Telescope (JCMT) and radio data from Westerbork Synthesis Radio Telescope (WSRT) were also obtained in the NEP-Deep field (see also Wada et al. 2008). Thus this multiband AKARI NEP-Deep field data set provides a unique opportunity to study star formation in infrared luminous galaxies.

Deep ancillary optical and NIR data covering the entire NEPDeep region are essential for finding counterparts of sources detected with AKARI. In addition, multiwavelength data are needed for accurate photometric redshift estimation, especially in covering the rest-frame wavelength range around the $4000 \AA$ break. Many studies have been made of the optical counterparts of the AKARI sources. Takagi et al. (2012) used the Subaru/Suprime-cam to obtain deep optical images (28.4 mag for $B$-band, $28.0 \mathrm{mag}$ for $V, 27.4 \mathrm{mag}$ for $R, 27.0 \mathrm{mag}$ for $i^{\prime}$, and $26.2 \mathrm{mag}$ for $z^{\prime}$ in the $A B$ magnitude system), but these data only cover one half of the NEP-Deep area. Hwang et al. (2007) observed the entire NEP-Deep field with the Canada France Hawaii Telescope (CFHT) in the $g^{\prime}, r^{\prime}, i^{\prime}$, and $z^{\prime}$-bands with limiting magnitudes of 26.1, 25.6, 24.7, and 24.0 mag $(4 \sigma)$ with 1 arcsec diameter apertures. However, these images have insensitive areas resulting from gaps between CCD chips. Imai et al. (2007) observed the area with KPNO-2.1 m/FLAMINGOS in the $J$ - and $K_{\mathrm{s}}$-bands, but the $3 \sigma$ detection limits were shallow (21.85 mag and $20.15 \mathrm{mag}$ in the $J$ and $K_{\mathrm{s}}$-bands, respectively). Moreover,

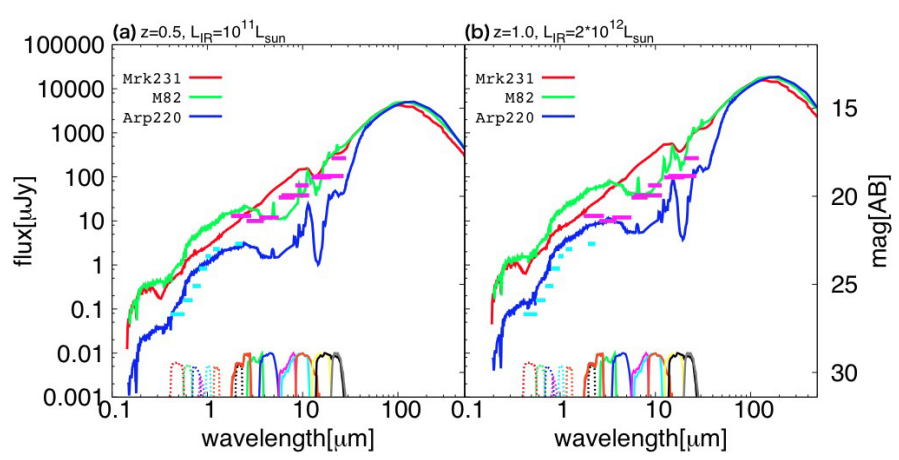

Fig. 1. Example of galaxies SEDs. Left panel shows the SEDs with redshift of 0.5 with infrared luminosity of $10^{11} L_{\odot}$, while the right panel is for the SEDs with redshift of 1.0 and an infrared luminosity of $2 \times 10^{12} L_{\odot}$. Red, green, and blue solid lines represent SEDs of Mrk231, M82, and Arp220. Thick horizontal lines in pink and sky blue show the $5 \sigma$ detection limits of AKARI $N 2-L 24$ filters and ground-based $g^{\prime}-$ $K_{\mathrm{s}}$ band $4 \sigma$ limiting magnitudes that we observed (see Table 2). Filter response curves of CFHT/MegaCam, WIRCam, and AKARI/IRC are shown at the bottom of the panels. Red, green, blue, pink, cyan, orange, and black dotted lines represent $g^{\prime}, r^{\prime}, i^{\prime}, z^{\prime}, Y, J$, and $K_{\mathrm{s}}$, while red, green, blue, pink, cyan, yellow, black, orange, and gray solid lines are AKARI/IRC N2 - L24 bands.

these data cover only about one third of the NEP-Deep field $\left(25^{\prime} \times 30^{\prime}\right)$.

Figure 1 is an example of SEDs for the star-forming galaxies Mrk231, M82, and Arp220 if their redshifts are at $z=0.5$ with infrared luminosity of $10^{11} L_{\odot}$ (left) or $z=1.0$ with infrared luminosity of $2 \times 10^{12} L_{\odot}$. To optically identify Arp220 at $z=1$ (blue SED), which can be detected by AKARI, 26-23 mag [AB] for $g^{\prime}-z^{\prime}$ bands and 22-23 mag [AB] for NIR bands are required. In this paper, we present deep optical $g^{\prime}, r^{\prime}, i^{\prime}$, and $z^{\prime}$ and NIR $Y, J$, and $K_{\mathrm{s}}$ imaging data in the NEP-Deep field using MegaCam (Boulade et al. 2003) and the Wide-field InfraRed Camera (WIRCam; Puget et al. 2004) on the CFHT (Fig. 2). We also combine existing $u^{*}$-band data (PI: S. Serjeant; Takagi et al. 2012) to provide a band-merged catalog based on the $z^{\prime}$-band. The catalog is used to analyze the AKARI NEP sources.

This paper is organized as follows. We describe our observations and data reduction procedures in Sect. 2 and source extraction in each band in Sect. 3. We present our band-merged catalog in Sect. 4. In Sect. 5, we describe our method of star-galaxy separation based on color-color criteria and the SExtractor stellarity index. We calculated photometric redshifts for the sources in our catalog by spectral energy distribution (SED) fitting, which is presented in Sect. 6. Section 7 provides the properties of AKARI sources identified by their optical counterparts in our catalog. We summarize our results in the final section.

\section{Observation and data reduction}

\subsection{CFHT/MegaCam (optical: $g^{\prime}, r^{\prime}, i^{\prime}$, and $z^{\prime}$-bands)}

We obtained optical $\left(g^{\prime}, r^{\prime}, i^{\prime}\right.$, and $\left.z^{\prime}\right)$ imaging of nearly the entire NEP-Deep field with CFHT/MegaCam at the telescope prime focus. The observations were carried out in queue mode, spread over 22 photometric nights from 2011 April 27 to 2011 September 4, resulting in a total of 166 frames (PI: T. Goto). MegaCam is a wide-field imager composed of $362048 \times$ 4612 pixel CCDs, covering a full $1 \times 1 \mathrm{deg}^{2}$ field of view with a pixel scale of 0.187 arcsec. The MegaCam $g^{\prime}, r^{\prime}, i^{\prime}$, and $z^{\prime}$-band 


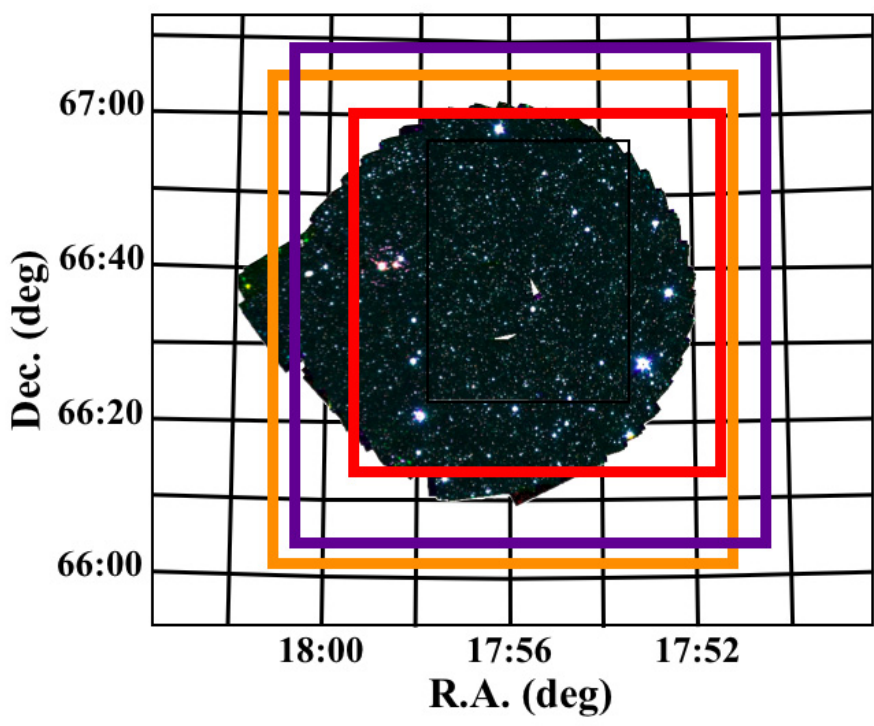

Fig. 2. Coverage of various surveys in the NEP-Deep field. The color image in the middle is the three-color NIR image made with the IRC data (blue $-2 \mu \mathrm{m}$, green $-3 \mu \mathrm{m}$, red $-4 \mu \mathrm{m}$ ). The orange box is the survey coverage of MegaCam $g^{\prime}, r^{\prime}, i^{\prime}$, and $z^{\prime}$, and the red box is that of WIRCam $Y, J$, and $K_{\mathrm{s}}$. The purple box represents the MegaCam $u^{*}$-band observation field (Takagi et al. 2012).

filters follow the original Sloan Digital Sky Survey (SDSS; York et al. 2000) filters ${ }^{1}$.

The preprocessing, including bias subtraction and flatfielding of raw images, was carried out using the Elixir pipeline (Magnier \& Cuillandre 2004). Elixir is a collection of programs and databases, specialized for MegaCam data. By stacking the Elixir-processed images, we produced a final mosaicked image using the AstrOmatic softwares including WeightWatcher ${ }^{2}$ (Marmo \& Bertin 2008) for creating a weight map, SExtractor ${ }^{3}$ (Bertin \& Arnouts 1996) for the extraction and photometry of sources, SCAMP ${ }^{4}$ (Bertin 2006) for astronomical calibration with 2MASS as an astrometric reference catalog, and modified SWarp 5 (Bertin et al. 2002) with a three-sigma clipping function $^{6}$ for stacking images. The images were scaled to have a photometric zero point of 30.000 in $A B$ magnitudes. Before the stacking, images were sampled again using a Lanczos-3 six-tap filter with a 128-pixel mesh by SWarp. The lefthand panel of Fig. 3 shows the FWHM distribution of the individual images used for the stacking measured by the Elixir. We can see that the FWHM ranges for $r^{\prime}-z^{\prime}$-bands are $0.60-1.20$ and the range for the $g^{\prime}$ band is slightly worse $(0.75-1.25)$ compared with the others. The FWHMs of final stacked images for $r^{\prime}-z^{\prime}$ bands are $\sim 0.85-0.9$ arcsec, and 0.96 for the $g^{\prime}$ band.

In this work we have carefully excluded a false source contamination of our final catalog. During this data reduction, we removed peaked isolated noise using the normalized median absolute deviation (MAD; Beers et al. 1990). We considered 15 pixels surrounding each pixel in each image, and calculated median and MAD values. If the original pixel value deviated by

\footnotetext{
http://www3.cadc-ccda.hia-iha.nrc-cnrc.gc.ca/ megapipe/docs/filters.html

2 http://www .astromatic.net/software/weightwatcher

3 http://www.astromatic.net/software/sextractor

4 http://www.astromatic.net/software/scamp

5 http://www.astromatic.net/software/swarp

6 This version of SWarp is modified to have 3-sigma clipping function by S. Foucaud (priv. comm.).
}
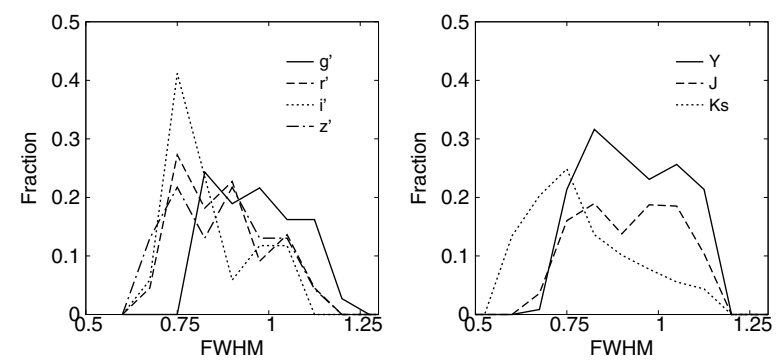

Fig. 3. FWHM distribution of each flame we used in the optical (left) and NIR (right). The solid, dashed, dotted, and dashed-dotted lines in the left panel show the $g^{\prime}, r^{\prime}, i^{\prime}, z^{\prime}$ bands, respectively, while the solid, dashed, and dotted lines in the right panel represent the $Y, J, K_{\mathrm{s}}$ bands.

more than 3 MAD from the median value, it was regarded as bad and was replaced with the median value. Since fake sources lying astride over a few pixels or along artificial structures close to bright sources cannot be removed by the MAD method, we used those weight maps during source extraction by SExtractor (see Sect. 3.1) and carried out a band-merging procedure (see Sect. 4.2) to reduce the false source contaminations.

\subsection{CFHT/WIRCam (NIR: $Y, J$, and $K_{s}$-bands)}

We used the CFHT/WIRCam to obtain deep NIR $\left(Y, J\right.$, and $\left.K_{\mathrm{s}}\right)$ images at the NEP-Deep field. WIRCam uses four $2048 \times 2048$ HAWAII2RG CCD arrays. The camera provides 0.3 arcsec per pixel sampling and the close packing enables coverage of an almost contiguous field of view of $20.5^{\prime} \times 20.5^{\prime}$. Observations in $Y, J$, and $K_{\mathrm{s}}$-bands were conducted in queue mode spread over 19 photometric nights from 2010 March 31 to 2010 June 29, resulting in a total of 2009 frames (PI: T. Goto). We observed four positions with dithering, completely covering a $47^{\prime} \times 44^{\prime}$ field of view in each band.

The preprocessing, i.e., removal of the instrumental signature and astrometry, for raw WIRCam images was carried out with the 'I'iwi ${ }^{7}$ preprocessing pipeline. In general, photometric calibration by SCAMP is based on both internal pairing among frames of overlapping regions with SExtracted source catalogs of WIRCam itself and external pairing with reference catalogs with SExtracted source catalogs of WIRCam. However, we found the internal pairing method did not work well when crosschecking the uniformity of the photometric zero point across the SWarp stacked images by using the SCAMP photometric solution. Therefore, in this paper, we only used the external pairing method. Photometric calibration of the $J$ and $K_{\mathrm{s}}$-band observations were performed by matching to the corresponding 2MASS catalog, and the weighted images were then stacked using the modified SWarp. We compared 2MASS sources with photometric errors of less than $0.05 \mathrm{mag}$, i.e., $J<16.48 \mathrm{mag}$ and $K_{\mathrm{s}}<$ $15.90 \mathrm{mag}$, with the extracted sources of WIRCam. We found the zero point and its uniformity within the $47 \times 44 \operatorname{arcmin}^{2}$ area for the $J$ and $K_{\mathrm{s}}$-bands to be $25.964 \mathrm{mag}(0.072 \mathrm{mag})$ and $26.927 \mathrm{mag}(0.073 \mathrm{mag})$, respectively.

Since the 2MASS catalog does not have $Y$-band magnitudes, we did the photometric calibration of the $Y$-band by comparing it with the predicted $Y$-band magnitudes estimated by SED fitting of stars. For the purpose of creating the predicted $Y$-band magnitude catalog as the external reference, we first created a band-merged catalog (see Sect. 4) and selected stars with a

\footnotetext{
http://cfht.hawaii.edu/Instruments/Imaging/WIRCam/ IiwiVersion1Doc.html
} 


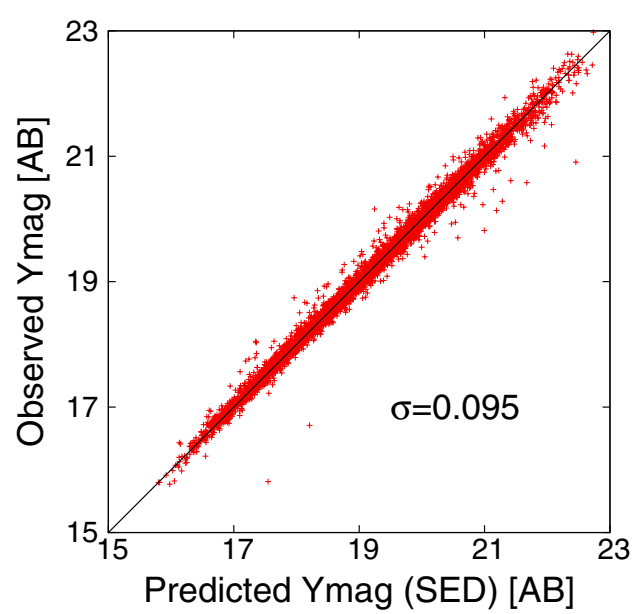

Fig. 4. Comparison between the observed $Y$-band magnitude and predicted $Y$-band magnitude estimated by SED fitting of stars. From this comparison, the photometric zero point and the RMS in the $Y$-band are $25.715 \mathrm{mag}$ and $0.095 \mathrm{mag}$ for $Y<23 \mathrm{mag}$, respectively.

stellarity index given by SExtractor, CLASS_STAR parameter in the $z^{\prime}$-band image, and appropriate $u^{*}-J$ and $g^{\prime}-K_{\mathrm{S}}$ colors (see Sect. 5). We calculated the predicted $Y$-band magnitude based on the $\chi^{2}$ fitting analysis of the SED using a publicly available LePhare code (Ilbert et al. 2006; Arnouts et al. 2007; Ilbert et al. 2009) with 154 star templates, which include main sequence stellar SEDs from Pickles (1998) and white dwarf SEDs from Bohlin et al. (1995). For the SED fitting, we input the photometric data sets from $u^{*}$ to $K_{\mathrm{s}}$-bands omitting the $Y$-band. Then we calibrated the photometry with the predicted $Y$-band magnitude as the external reference catalog of SCAMP and made the final images with the modified SWarp. In Fig. 4, we compared the observed $Y$-band magnitudes from the final image with the predicted magnitudes based on the SED fitting for the selected stars. The figure shows that both values are agreed with each other. We obtained the photometric zero point and the root mean square (RMS) in the $Y$-band as $25.715 \mathrm{mag}$ and 0.095 ( $Y<23 \mathrm{mag}$ ).

Before the final stack, we samplied WIRCam images again using the Lanczos-3 six-tap filter with a 128-pixel mesh by Swarp, as well as MegaCam imagers. And then we adopted the threshold of $F W H M<1.2$ arcsec estimated by SExtractor to remove the bad quality images. The FWHM distributions we used for the final stacked images are shown in the righthand panel of Fig. 3, and the seeing of the final stacked images of $Y, J$, and $K_{\mathrm{s}}$-bands are all $\sim 0.7-0.8$ arcsec.

\section{Procedure for source extraction}

\subsection{Source extraction}

For detecting objects in MegaCam and WIRCam images, we used SExtractor. The seeing is not homogeneous across bands (see Fig. 3 and in Table 2). To carry out the photometry for each band with optimal aperture, we ran SExtractor with each band image individually with the same parameter set. The SExtractor parameter set for all bands is summarized in Table 1. A 7 pix $\times 7$ pix Gaussian point spread function (PSF) convolution filter with $F W H M=3.0$ pixels was used to help detect faint sources. Fluxes of extracted sources were measured using SExtractor with elliptical apertures of 2.5 times of the Kron radius (Kron 1980), i.e., SExtractor's MAG_AUTO. We used weight maps created during the SWarp process to avoid false detections. Figure 5 is an example of weight maps (left: MegaCam
Table 1. Parameters of SExtractor.

\begin{tabular}{lc}
\hline \hline Name & Value \\
\hline DETECT_THRESH & 1.5 \\
DETECT_MINAREA & 5 \\
DEBLEND_MINCONT & 0.001 \\
FILTER_NAME & gauss_3.0_7x7.conv \\
WEIGHT_TYPE & MAP_WEIGHT \\
\hline
\end{tabular}

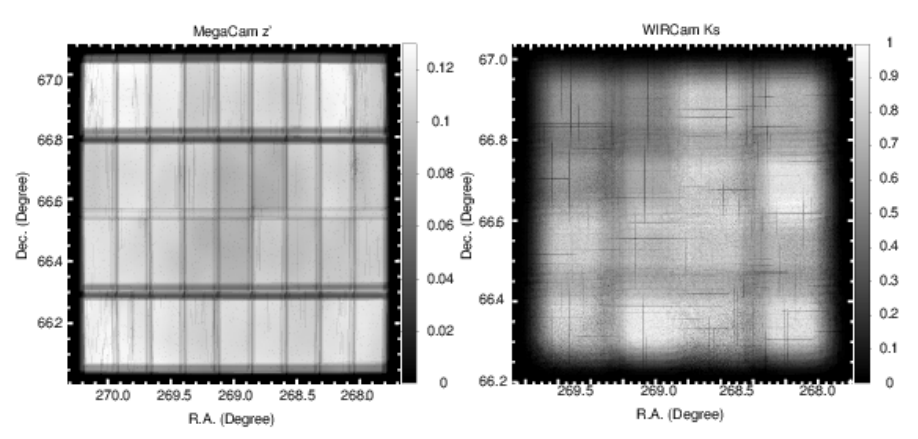

Fig. 5. Examples of weight maps for MegaCam $z^{\prime}$-band (left) and $K_{\mathrm{s}}$-band (right). The pixel values are inversely proportional to the local variance of the image, and the areas of the pixel value of 0 are the regions without data. Crosses of clearly less weighted areas in MegaCam data are present owing to the gaps between detectors, while WIRCam data are relatively homogeneous.

$z^{\prime}$; right: WIRCam $K_{\mathrm{s}}$ ). Weight maps of the other MegaCam bands are very similar to that of the $z^{\prime}$-band and those of the other WIRCam bands are almost the same as that of the $K_{\mathrm{s}}$-band. The MegaCam $z^{\prime}$-band weight map shows four less weighted regions in a cross direction and eight minor less weighted regions in the longitudinal direction due to the detector gaps. In comparison, the WIRCam $K_{\mathrm{s}}$-band weight map looks nicely uniform with no distinct less weighted gaps. Data at the less weighted area are relatively noisier than in the other area, and noise causes false detections. Weight maps affect error estimates and reduce the number of false detections from the noisy region.

From the extracted source catalog for each band, we removed sources with a photometric flag of four or larger from the detected source list because these sources can be unreliable, indicating that at least one pixel is saturated, or close to the image boundary, or both. The number of sources in the extracted catalog for each band is given in Table 2. Figure 6 (except the bottom right panel) shows number counts for all our seven-band data. Number counts from Hwang et al. (2007) for the NEPWide field and those from 2MASS data around the NEP region are overplotted in the figure as references of total (star + galaxy) number counts. We found that our total number counts are consistent with those surveys' results. We also calculated the number counts of stars at around NEP-region using a TRILEGAL galaxy model (Girardi et al. 2012) in each band, and then we subtracted it from the total number counts to estimate galaxy number counts. The number counts are compared with CFHTLS T0007 ${ }^{8}$ for MegaCam data and with the WIRCam Deep survey (Bielby et al. 2012) for the WIRCam $J$ and $K_{\mathrm{s}}$ bands. In the bright magnitude range, our galaxy number counts in $g^{\prime}$-band is slightly more than those from CFHTLS, while in the $z^{\prime}$ and $J$ bands, our results are slightly less than that of the comparison fields. These differences are about $15 \%$ and can be explained by

\footnotetext{
8 http://www.cfht.hawaii.edu/Science/CFHTLS/T0007/ CFHTLS_TQ007-TechnicalDocumentation.pdf
} 
Table 2. Summary of properties of the extracted source catalogs for each band.

\begin{tabular}{|c|c|c|c|c|c|c|c|}
\hline & \multicolumn{4}{|c|}{ MegaCam } & \multicolumn{3}{|c|}{ WIRCam } \\
\hline & $g^{\prime}$ & $r^{\prime}$ & $i^{\prime}$ & $z^{\prime}$ & $Y$ & $J$ & $K_{\mathrm{s}}$ \\
\hline FWHM (arcsec) & 0.96 & 0.88 & 0.84 & 0.86 & 0.80 & 0.81 & 0.66 \\
\hline Total integration time $[\mathrm{ks}]$ & 23.2 & 17.6 & 27.6 & 23.2 & 21.2 & 19.9 & 18.3 \\
\hline Detection number & 224,449 & 178,191 & 143,318 & 88,666 & 39.063 & 34,138 & 38,053 \\
\hline Limiting mag $[\mathrm{AB}]$ & 26.7 & 25.9 & 25.1 & 24.1 & 23.4 & 23.0 & 22.7 \\
\hline $50 \%$ Completeness $[\mathrm{AB}]$ & 26.1 & 25.4 & 24.9 & 23.7 & 22.8 & 22.4 & 22.3 \\
\hline MAG_APER - MAG_AUTO [AB] & 0.55 & 0.56 & 0.49 & 0.53 & 0.48 & 0.47 & 0.38 \\
\hline
\end{tabular}

Notes. Line (1) FWHM of PSF. Line (2) Integration time. Line (3) Number of detected sources in each band image. Line (4) Limiting magnitude in each band with a threshold of $4 \sigma$ detection within a 1 arcsec radius. Line (5) 50\% completeness limit. Line (6) Magnitude difference between MAG_APER with 1 arcsec radius and MAG_AUTO for objects at $0.45<z<0.55$.
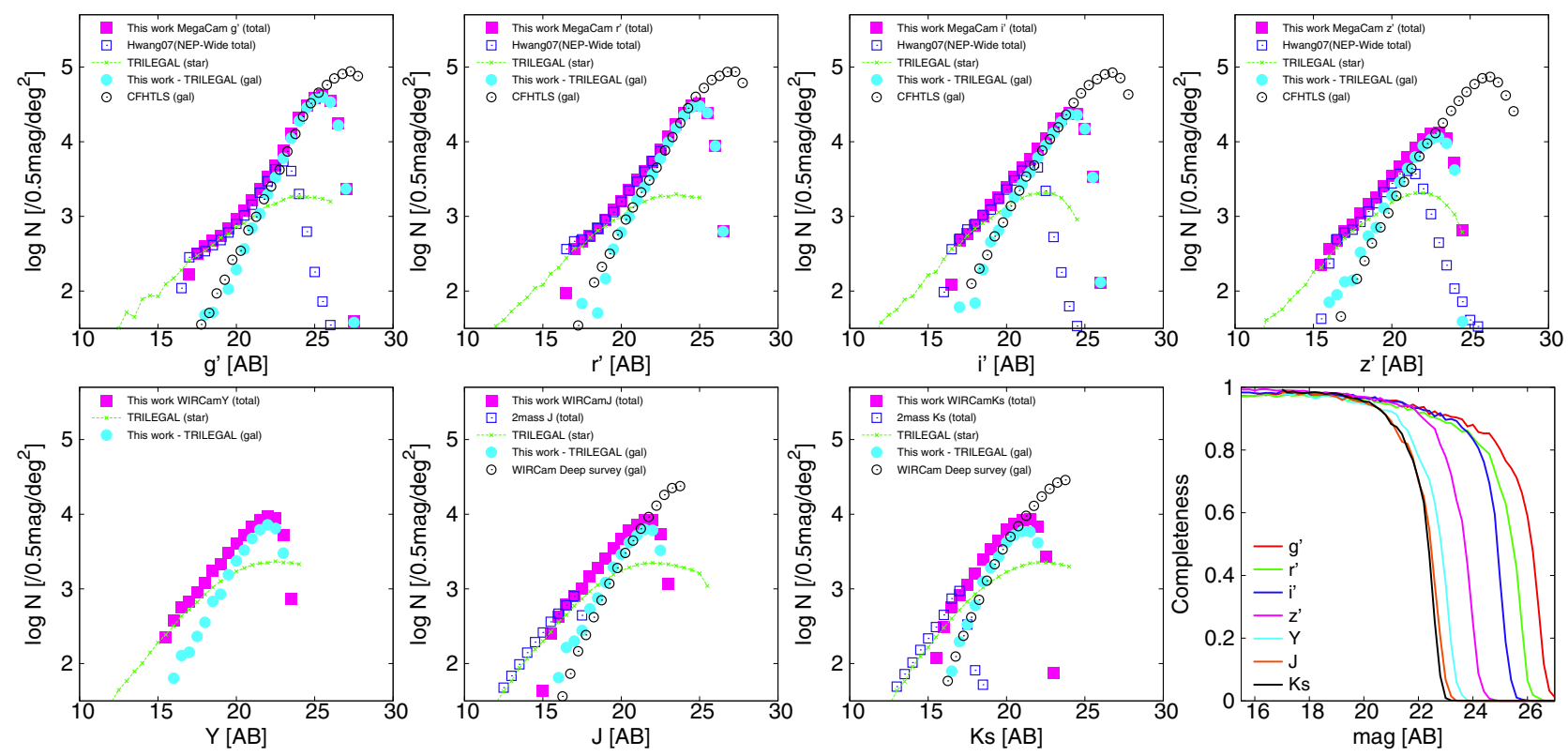

Fig. 6. Number counts (all panels but right bottom one) and completeness (right bottom panel) for $g^{\prime}, r^{\prime}, i^{\prime}, z^{\prime}, Y, J$, and $K_{\mathrm{s}}$-bands. In the number count panels, pink filled squares represent total number counts in the NEP-Deep field (this work), and green lines show a number count of stars around NEP-Deep Field using a TRILEGAL galaxy model (Girardi et al. 2012). Cyan filled circles represent number counts of galaxies, estimated with total minus stellar number counts. As references, we overplotted total number counts at NEP-Wide field (blue squares, Hwang et al. 2007) and galaxy number counts from CFHTLS T0007 data for optical bands, while total number counts from 2MASS and galaxies one from WIRCam Deep survey (Bielby et al. 2012) for NIR. In the bottom right panel, red, green, blue, pink, cyan, orange, and black lines represent the completeness of $g^{\prime}-K_{\mathrm{s}}$.

an uncertainly in the TRILEGAL models. At the fainter magnitude range, where the effect from star number counts is weak, our results and their comparisons are in excellent agreement with each other.

\subsection{Detection limits and completeness}

We determined the $4 \sigma$ detection limits over a circular aperture of 1 arcsec radius. The detection-limit changes depending on the source position on the mosaicked images since the exposure map is not uniform. We present the median values over the entire field for all MegaCam and WIRCam bands in Table 2. The limiting magnitudes are 26.7, 25.9, 25.1, and 24.1 mag for MegaCam $g^{\prime}$, $r^{\prime}, i^{\prime}$, and $z^{\prime}$ bands, and 23.4, 23.0, 22.7 mag for WIRCam $Y, J$, $K_{\mathrm{s}}$ bands, respectively. The completeness of each band was estimated via simulations with artificial sources. We produced 2000 artificial sources per 0.2 mag with the averaged radial profile of 20 point sources in each band. We separated the artificial sources by at least 60 pixels from each other. We ran SExtractor on each image with the artificial sources by utilizing exactly the same extraction parameters as for the original images. The success of detecting those artificial sources is judged by the source location (within 1 arcsec from the true location) and magnitude (within $0.5 \mathrm{mag}$ from the true magnitude). The $50 \%$ completeness limits are listed in Table 2, and the completeness curves are shown in righthand panel of Fig. 6 as a function of magnitude. The completeness curves show that the detection probability begins to drop rapidly at around $90 \%$ value, and the magnitude difference between $90 \%$ and $10 \%$ completeness is about 2 mag.

The limiting magnitudes listed in Table 2 are actually for point sources because the values represent the $4 \sigma$ detection within a 1 arcsec aperture radius. Over $99 \%$ of the flux of a point source is included within the aperture $(F W H M=0.8-0.9$ arc$\mathrm{sec}$ ), while an extended galaxy at low redshift with magnitude equal to the limiting magnitude cannot be detected with $4 \sigma$ since such galaxies are spatially resolved in our images. We measured the difference between the 1 arcsec radius aperture magnitude (MAG_APER) and the total magnitude (MAG_AUTO). In 
Table 3. Accuracy of astrometry in arcsec.

\begin{tabular}{crrrrr}
\hline \hline \multirow{2}{*}{ Band } & \multicolumn{2}{c}{$\Delta$ RA } & & \multicolumn{2}{c}{$\Delta$ Dec } \\
\cline { 2 - 3 } \cline { 6 - 6 } & \multicolumn{1}{c}{ offset } & RMS & & offset & RMS \\
\hline$z^{\prime}$ vs. 2MASS & 0.0018 & 0.2067 & & 0.0129 & 0.2420 \\
$u^{*}$ vs. $z^{\prime}$ & -0.0043 & 0.1114 & & 0.0098 & 0.1438 \\
$g^{\prime}$ vs. $z^{\prime}$ & -0.0041 & 0.0733 & & 0.0107 & 0.0821 \\
$r^{\prime}$ vs. $z^{\prime}$ & -0.0024 & 0.0645 & & 0.0032 & 0.0641 \\
$i^{\prime}$ vs. $z^{\prime}$ & -0.0019 & 0.0591 & & 0.0016 & 0.0583 \\
$Y$ vs. $z^{\prime}$ & -0.000003 & 0.0613 & & 0.0002 & 0.0630 \\
$J$ vs. $z^{\prime}$ & 0.0002 & 0.0620 & & 0.0003 & 0.0626 \\
$K_{\text {s vs. } z^{\prime}}$ & 0.0003 & 0.0623 & & -0.0001 & 0.0628 \\
\hline
\end{tabular}

Sect. 6, we calculate the photometric redshift via SED fitting and find the peak redshift distribution of our data to be $z \sim 0.5$. We then compared the MAG_APER with MAG_AUTO for galaxies at $z \sim 0.5(0.45<z<0.55)$ as a typical example in our data. The differences in all the images is around $0.38-0.56 \mathrm{mag}$. We, therefore, note that a limiting magnitude for extended sources is typically brighter by $\sim 0.5 \mathrm{mag}$.

\section{Band-merged catalog based on $z^{\prime}$ band}

In this section, we describe the processes of making the bandmerged catalog. One of our aims in this work is to construct a $z^{\prime}$-band-based band-merged catalog and find counterparts for AKARI infrared sources (Murata et al. 2013). We use optical data as a baseband for the band-merged catalog since the optical data cover the AKARI NEP-Deep field wider than the NIR ones. Since a redder band is much more useful for identifying AKARI sources because bluer bands can be affected by heavy dust extinction, the $z^{\prime}$ band is adopted as the baseband, although it is shallower than the bluer bands.

Since $u^{*}$-band photometric data are important for the photometric redshift estimation in Sect. 6, we merged not only the newly obtained MegaCam 4 bands and WIRCam 3 bands, but also the $u^{*}$-band catalog constructed by Takagi et al. (2012). This $u^{*}$-band image is centered at $\mathrm{RA}=17^{\mathrm{h}} 55^{\mathrm{m}} 24^{\mathrm{s}}$, Dec $=$ $+66^{\circ} 37^{\prime} 32^{\prime \prime}$ [ J2000] and has a limiting magnitude of $24.6 \mathrm{mag}$ $[5 \sigma ; \mathrm{AB}]$. Details of the source extraction and the photometry for the $u^{*}$ band are described in Sect. 2.2.2 of Takagi et al. (2012).

\subsection{Astrometric accuracy}

We cross-matched sources in all the bands to create the bandmerged catalog based on the $z^{\prime}$-band and checked the astrometric accuracy by examining the coordinate offset with respect to 2MASS and our other band catalogs. Counterparts in each catalog for $z^{\prime}$-band sources were searched within a 1 arcsec radius. We found 2334 counterparts of 2MASS sources. The spatial distribution of the offset, mean offsets and RMS offset, are 0.013 arcsec and 0.32 arcsec, respectively. For the other bands, the RMS for the $u^{*}$ band is 0.18 arcsec, and 0.08-0.11 arcsec in the other bands. The large RMS in the $u^{*}$ band is probably caused by a slightly degraded PSF. All the offsets and the RMS offsets are summarized in Table 3, and Fig. 7 shows examples of the relative positions between the $z^{\prime}$ band and 2MASS, $u^{*}$, and $K_{\mathrm{s}}$ bands.
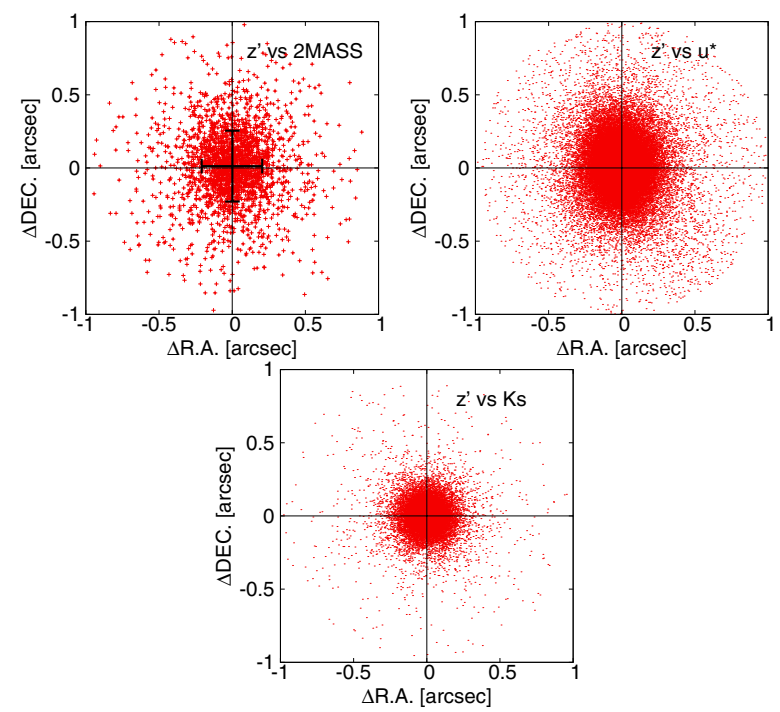

Fig. 7. Relative positions of the counterparts between the $z^{\prime}$-band and 2MASS, $u^{*}$, and $K_{\mathrm{s}}$-band catalogs. The error bars in the top left figure indicate the root mean square for each RA and Dec direction.

\subsection{Band merging}

To identify counterparts with cross-matching between $z^{\prime}$-band and the other bands, we adopted a 0.5 arcsec search radius that corresponds to three times the RMS of astrometric accuracy between the $z^{\prime}$ and $u^{*}$ bands, i.e., the pair that gave the lowest coordinate matching accuracy. This small search area size helped us minimize the chance coincidence rate (see Sect. 4.3).

After the source matching, we compiled all the catalogs into a single band-merged catalog based on the $z^{\prime}$-band sources. To ensure a robust catalog against false sources and transients (e.g., time variable objects and asteroids), we excluded sources with only $z^{\prime}$-band detection. As a result, the catalog includes 85797 out of $88666 z^{\prime}$-band sources. The number of matched sources between the $z^{\prime}$ band and the other bands are summarized in Table 4. In Table 5, we summarize the statistics of sources with multiple band detections for all eight bands in the optical and NIR together.

During the matching of the sources, we identified three types of counterpart identifications: (Case A) a single $z^{\prime}$-band source has a unique counterpart, (Case B) a single $z^{\prime}$-band source has multiple counterpart candidates, and (Case C) multiple $z^{\prime}$-band sources have one common counterpart candidate. Owing to the small searching area ( 0.5 arcsec radius), a significant number of the counterparts of $z^{\prime}$-band sources are categorized into Case A. We assigned the " 0 " flag to these unique pairs to indicate that the pair is reliable in our band-merged catalog. Cases B and C are both very rare for all bands $(<0.01 \%)$ except Case B in the $u^{*}$ band. We visually checked the $u^{*}$-band image and suspect that distorted PSF in the $u^{*}$ band causes these multiple detections of a single source. We treated Case B by summing fluxes of the multiple counterpart candidates and give them a flag " 1 ", while for Case $\mathrm{C}$ we listed the common source in other bands as a counterpart for both of the $z$-band sources with flag " 2 " in the catalog. The number of pairs found in those case are summarized in Table 4.

\subsection{False matching rate}

Reducing the risk of contamination by false sources is one of important points for catalog construction. For this purpose, we 
Table 4. Summery of counterpart matching.

\begin{tabular}{ccccccccc}
\hline \hline & \multicolumn{4}{c}{ MegaCam } & & \multicolumn{3}{c}{ WIRCam } \\
\cline { 2 - 4 } \cline { 7 - 9 } & $u^{*}$ & $g^{\prime}$ & $r^{\prime}$ & $i^{\prime}$ & & & $J$ & $K_{\mathrm{s}}$ \\
\hline Total & 55145 & 75961 & 79454 & 83295 & & 34162 & 31002 & 31634 \\
Case A (flag=0) & 55063 & 75959 & 79452 & 83286 & & 34160 & 31000 & 31634 \\
Case B (flag=1) & 82 & 0 & 2 & 5 & & 0 & 0 & 0 \\
Case C (flag=2) & 0 & 2 & 0 & 4 & & 2 & 2 & 0 \\
\hline
\end{tabular}

Notes. Line (1) represents the total number of $z^{\prime}$-band sources with counterparts in other bands. The number of $z^{\prime}$-band sources classified into Cases A, B, and C, are summarized in lines (2)-(4), respectively, for each band.

Table 5. Number of sources with multiple band detections.

\begin{tabular}{ccccccccc}
\hline \hline \# of detected bands & 2 & 3 & 4 & 5 & 6 & 7 & 8 & total \\
\hline \# of sources & 2583 & 3725 & 17214 & 28727 & 6108 & 8550 & 18890 & 85797 \\
fraction & 0.03 & 0.04 & 0.20 & 0.34 & 0.07 & 0.10 & 0.22 & 1 \\
\hline
\end{tabular}

cleaned up bad pixels (Sect. 2), used weight maps during extracting sources in each band image individually (Sect. 3.1), and cross-matched sources among bands and excluded sources detected only in the $z^{\prime}$ band (Sect. 4.2). However, there is a low, but non-zero possibility of false matching in the merged catalog. In this section we estimate the false matching rate in the catalog.

First, we estimated the false detection rate in each image. We multiplied each image by -1 to make a positive-negative reversed image, and then ran SExtractor on a negative image with the same parameters as for a positive image to detect false sources. The main locations of the false sources in the negative image are along the radial structures of bright stars and at edges of the image. The number of detections in the negative image for each band are summarized in Table 6. In the $z^{\prime}$-band case, we detected 88666 and 943 sources in the positive and negative images, respectively. Thus the false detection rate for $z^{\prime}$-band is $\sim 1.06 \%$. In our worst case, the $r^{\prime}$-band, the false detection rate is $\sim 1.65 \%$.

Second, we estimated a false-false matching rate between $z^{\prime}$ and other bands' spurious sources in the negative images with a 0.5 arcsec search radius. Even in the worst case, between $z^{\prime}$ and $g^{\prime}$-bands, the number of spuriously matched sources is only 15 $(15 / 943 \sim 1.60 \%)$. This low percentage is probably due to the fairly tight matching radius criterion.

Next, we measured a real-false matching rate between the sources in the positive $z^{\prime}$-band image and false sources in other bands' negative images. Even in the worst case, $z^{\prime}$ and $u^{*}$ bands, the rate is very low $(\sim 0.002 \%)$. The results of false matching are also shown in Table 6. Surprisingly, the real-false matching rate is less than the false-false matching rate in all bands except the $u^{*}$ band. This is because most false sources detected in the negative images are located around strong artifacts around bright stars, and the sky noise itself in each image randomly created less spurious sources. These results suggest that the real-false matching rate is extremely low in the final catalog when we use the 0.5 arcsec radius search area, nevertheless many of the faint sources appear around the artifacts and the edges of images.

Finally, we calculated the probability of chance coincidences between the real sources. If their source positions are completely uncorrelated and randomly distributed, the chance coincidence rate can be calculated with $n \times S$, where $n$ is number density of images and $S$ is the search area. In the case of the $g^{\prime}$-band, $n$ is 224450 per square degree and $S$ is $\pi \times(0.5 \operatorname{arcsec})^{2}$, giving a predicted chance coincidence of $1.36 \%$. However these positions
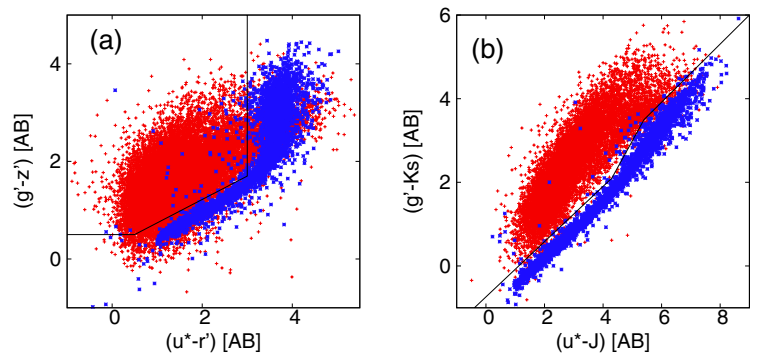

Fig. 8. (Left) $u^{*}-r^{\prime}$ versus $g^{\prime}-z^{\prime}$ diagram. Objects classified as stars by SExtractor (CLASS_STAR > 0.95) are plotted in blue and objects classified as galaxies (CLASS_STAR $<0.2)$ are in red. The solid line is the boundary separating the point sources from extended sources that Hwang et al. (2007) found. (Right) $u^{*}-J$ versus $g^{\prime}-K_{\mathrm{s}}$ color diagram. The same colors are used. The solid line is the boundary between the point sources and the extended sources as defined in the text.

are actually correlated when the sources are detected in the same field of view, because a source at a given band should be present in the other bands. Therefore the real chance coincidence rate in the final catalog is expected to be much lower than the predictions. We thus conclude that the false matching probability is negligibly low.

\section{Star - galaxy separation}

In this section, we attempt to separate stars securely from galaxies using the stellarity index of SExtractor (CLASS_STAR) and color. CLASS_STAR results from a supervised neural network that is trained to perform a star/galaxy classification with values between 0 and 1 . Sources with CLASS_STAR $>0.95$ (as stars) and those with the value $<0.2$ (as galaxies) in the catalog are plotted separately in the $u^{*}-r^{\prime}$ versus $g^{\prime}-z^{\prime}$ color plane (left panel of Fig. 8). The figure shows that they are clearly separated with some small fraction of exceptions by a three-straight-line boundary connecting $\left(u^{*}-r^{\prime}, g^{\prime}-z^{\prime}\right)=(-1.0,0.5),(0.5,0.5)$, $(3.0,1.7)$, and $(3.0,5.0)$ (Hwang et al. 2007). However, contamination by extended sources becomes larger at $u^{*}-r^{\prime}>3$, which is most likely due to red galaxies at $z<0.4$ (Fig. 1 of Strateva et al. 2001). In contrast, we found that the $u^{*}-J$ versus $g^{\prime}-K_{\mathrm{s}}$ color diagram (right panel of Fig. 8) enables us to separate stars from galaxies with less contamination. The boundary of these regions can be drawn by the combination of straight 
Table 6. Summary of chance coincidence rate in the final catalog.

\begin{tabular}{crrrrr}
\hline \hline Band & Positive & \multicolumn{1}{c}{ False } & \# of false-false match & \# of real-false match & Predicted \\
\hline$u^{*}$ & 149,168 & $1,475(0.99 \%)$ & $0(0.00 \%)$ & $2(0.002 \%)$ & $0.90 \%$ \\
$g^{\prime}$ & 224,449 & $2,361(1.05 \%)$ & $15(1.60 \%)$ & $1(0.001 \%)$ & $1.36 \%$ \\
$r^{\prime}$ & 178,191 & $2,944(1.65 \%)$ & $13(1.38 \%)$ & $1(0.001 \%)$ & $1.08 \%$ \\
$i^{\prime}$ & 143,318 & $911(0.64 \%)$ & $9(0.95 \%)$ & $0(0.00 \%)$ & $0.87 \%$ \\
$z^{\prime}$ & 88,666 & $943(1.06 \%)$ & $\mathrm{N} / \mathrm{A}$ & $\mathrm{N} / \mathrm{A}$ & $\mathrm{N} / \mathrm{A}$ \\
$Y$ & 39,063 & $456(1.17 \%)$ & $0(0.00 \%)$ & $0(0.00 \%)$ & $0.41 \%$ \\
$J$ & 34,138 & $183(0.54 \%)$ & $0(0.00 \%)$ & $0(0.00 \%)$ & $0.36 \%$ \\
$K_{\mathrm{S}}$ & 38,053 & $98(0.26 \%)$ & $0(0.00 \%)$ & $0(0.00 \%)$ & $0.40 \%$ \\
\hline
\end{tabular}

Notes. Column (1): band name. Column (2): the number of detected sources in the original images shown in Table 2. Column (3): the number (rate) of the detected sources in the negative image. Column (4): the number (rate) of matching between false sources in the negative images of the $z^{\prime}$-band and other bands within a 0.5 arcsec radius circle. Column (5): the number (rate) of matching between the real sources in the original $z^{\prime}$-band image and the false sources in the negative images of other bands within a 0.5 arcsec radius. Column (6): predicted probability of chance coincidence rate within a 0.5 arcsec radial area, assuming a spacial distribution of sources in the $z^{\prime}$-band and other bands are completely independent.
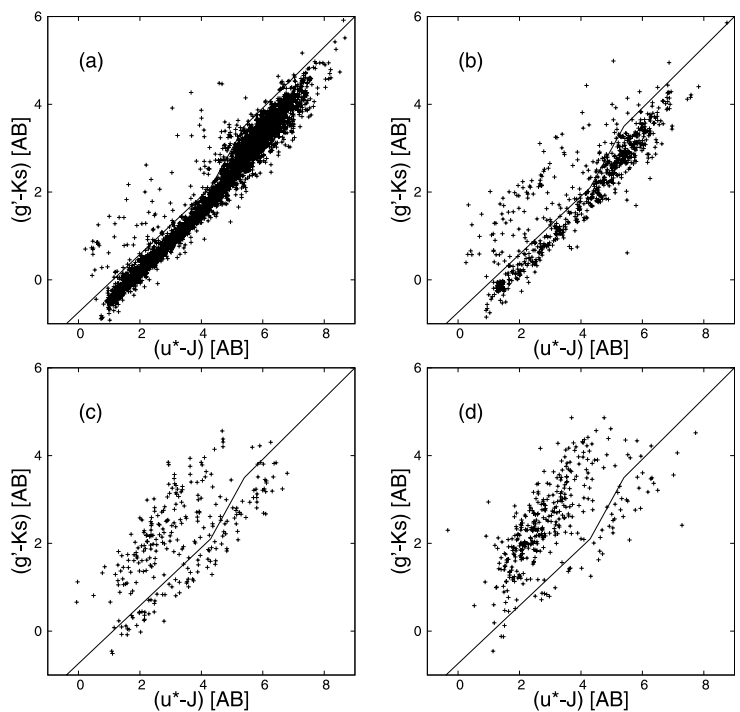

Fig. 9. $u^{*}-J$ versus $g^{\prime}-K_{\mathrm{s}}$ color-color diagram for different stellarity index ranges in the $z^{\prime}$-band. The (a)-(d) panels represent $0.9<$ CLASS_STAR $<1.0,0.8<$ CLASS_STAR $<0.9,0.7<$ CLASS_STAR $<0.8$, and $0.6<$ CLASS_STAR $<0.7$, respectively.

lines connecting at $\left(u^{*}-J, g^{\prime}-K_{\mathrm{S}}\right)=(-1.0,-1.4),(4.3,2.1)$, $(5.4,3.5)$, and $(9.0,6.0)$.

We also investigated the color dependence on the stellarity index ranges. We plotted sources with $0.9<$ CLASS_STAR $<$ $1.0,0.8<$ CLASS_STAR $<0.9,0.7<$ CLASS_STAR $<0.8$, and $0.6<$ CLASS_STAR $<0.7$ in the (a)-(d) panels of Fig. 9, respectively. The lines in all panels are the boundary shown in Fig. 8. The figure shows that most of the sources with CLASS_STAR > 0.8 in panels (a) and (b) fall under the boundary suggesting stars, while sources with CLASS_STAR $<0.7$ in panel (d) are mainly located above the boundary indicating extended sources. The sources at $0.7<$ CLASS_STAR $<0.8$ seem to be a mixture of the two classes. If we use only this color criterion and the stellarity index to separate stars from galaxies, quasars are likely to be misclassified as stars. To avoid this, we excluded sources bluer than $u^{*}-g^{\prime}=0.4$ from the star candidates (Finlator et al. 2000). In summary, the following criteria are for securely identifying stars: (1) $g^{\prime}-K_{\mathrm{S}}$ is bluer than the boundary in the $u^{*}-J$ versus $g^{\prime}-K_{\mathrm{S}}$ color diagram; (2) CLASS_STAR $>0.8$; and (3) $u^{*}-g^{\prime}>0.4$. 5441 star candidates satisfy those criteria in our catalog with a flag of "STAR". We note that the flagging of sources with at least one band non-detection in the $u^{*}, g^{\prime}, J$, and $K_{\mathrm{s}}$-bands is represented as “****" in the catalog because the color criteria cannot be used for them.

\section{Photometric redshift derivation}

\subsection{Photometric redshift computing with Le Phare}

We computed photometric redshifts $\left(z_{\mathrm{p}}\right)$ using the LePhare code (Ilbert et al. 2006, 2009; Arnouts et al. 2007). We used 62 galaxy templates and the 154 star templates that we used for predicting $Y$-band magnitude as the photometric reference during the earlier data reduction (Sect. 2.2). The galaxy templates are empirical ones from Coleman et al. (1980) with two starburst templates from Kinney et al. (1996). These templates are interpolated and adjusted to match the VIMOS VLT Deep Survey (VVDS) spectra better (Arnouts et al. 2007). For galaxy templates of Scd and later spectral types, we considered the dust extinction reddening. Since considerable changes in the extinction curve are expected from galaxy to galaxy, we used two extinction laws. We adopted an interstellar extinction law determined from starburst galaxies (Calzetti et al. 2000) and the Small Magellanic Cloud extinction law (Prevot et al. 1984) with varying $E(B-V)$ of $0.0,0.05,0.1$, $0.15,0.2,0.3,0.4$, and 0.5 . A broad absorption excess at $2175 \AA$ (UV bump) is sometimes needed to explain rest-frame UV flux in some starburst galaxies (Massarotti et al. 2001). Therefore we allowed an additional UV bump at $2175 \AA$ for the Calzetti extinction law if it produces a smaller $\chi^{2}$.

Systematic offsets are often found between the best fit SED templates and the observed apparent magnitudes in a given filter (Brodwin et al. 2006; Ilbert et al. 2006, 2009). Uncertainties in the zero point calibration of the photometric data, as well as imperfect knowledge of galaxy SEDs, are responsible for these offsets. They may produce biases in the photometric redshift measurements. In order to correct it, we computed the systematic offset $\left(s^{f}\right)$ for each filter, $f$, between the photometry in a given filter and the photometry for the best SED fitting at the fixed spectroscopic redshift $\left(z_{\mathrm{s}}\right)$ of the AKARI detected galaxies with the option AUTO_ADAPT in the LePhare algorithm. Excluding Type 1 AGN taken with Keck/DEIMOS (Takagi et al., in prep.), 483 sources from MMT/Hectospec and WIYN/HYDRA (Shim et al. 2013) are used as an input catalog for the training and estimation of the $z_{\mathrm{p}}$ accuracy. The all $z_{\mathrm{s}}$ of 483 galaxies were measured by using two or more emission lines or absorption lines. Many objects with $z_{\mathrm{s}}$ are distributed in the local universe at $z<1$ 


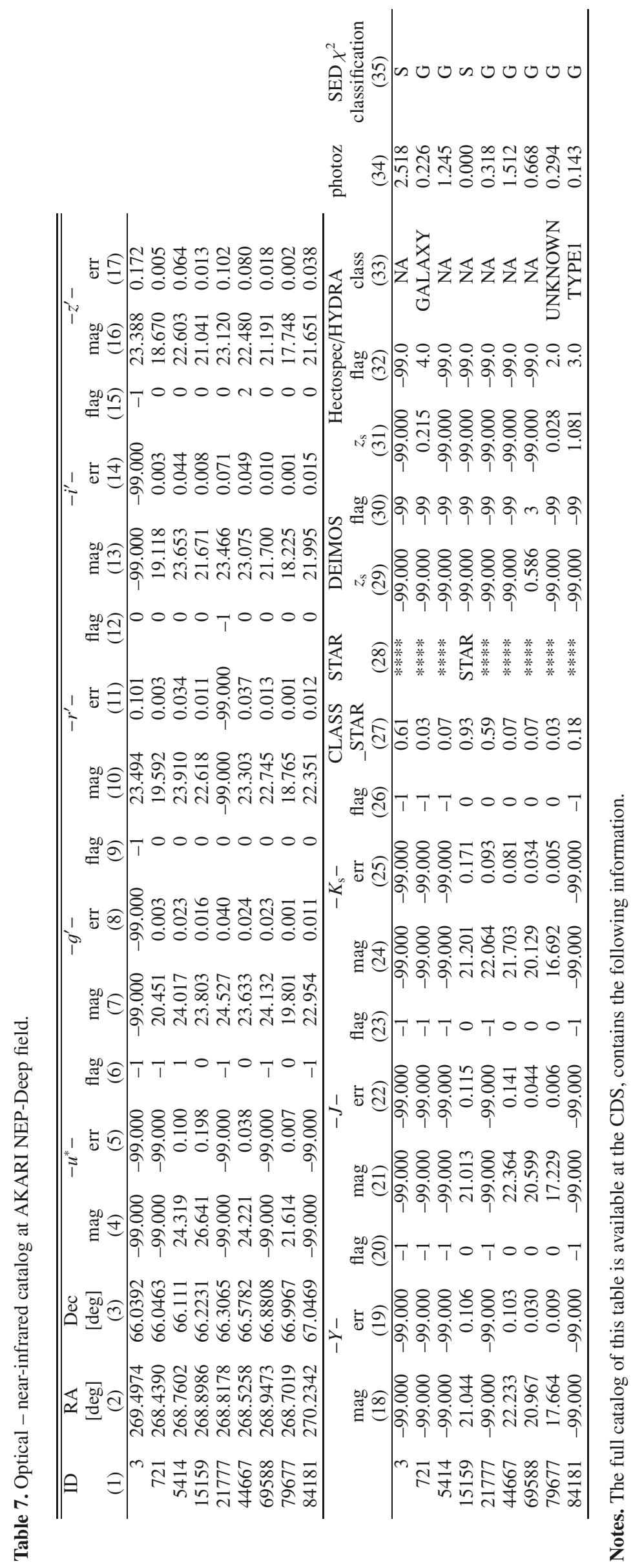




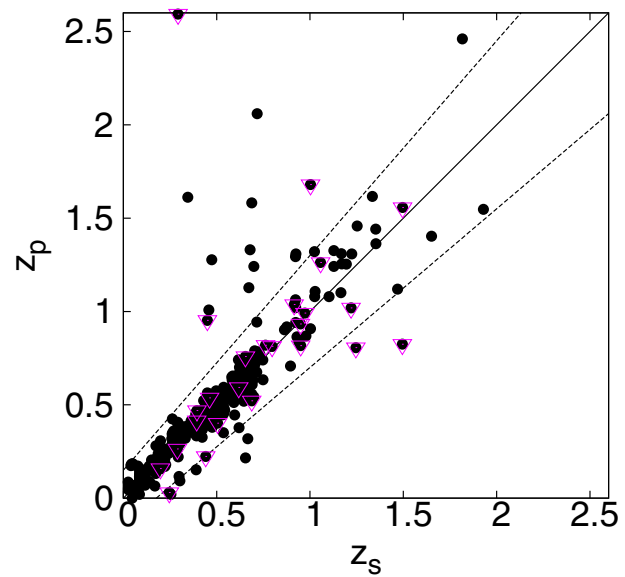

Fig. 10. Comparison between the photometric redshift $\left(z_{\mathrm{p}}\right)$ and the spectroscopic redshift $\left(z_{\mathrm{s}}\right)$. Pink triangles represent objects for which we found a second solution in the PDFz. For the sources with a single solution in the PDFz, an error of $z_{\mathrm{p}}$ is $\sigma_{\Delta z /(1+z)}=0.032$ and catastrophic error $(\Delta z /(1+z)>0.15)$ rate is $\eta=5.8 \%$ at $z<1$, while $\sigma_{\Delta z /(1+z)}=0.117$ and $\eta=16.6 \%$ for $z>1$.

(453 sources $\sim 94 \%$ ), and only $\sim 6 \%$ of the galaxy are at $z>1$. Iteratively, we then searched for the best set of corrections that minimized the offsets. Once found, the offset was applied to the SEDs when computing the photometric redshift for the entire catalog. The $z_{\mathrm{p}}$ is the redshift value that minimizes the merit function $\chi^{2}(z, T, A)$ :

$\chi 2=\Sigma_{f=1}^{N_{f}}\left(F_{\text {obs }}^{f}-A \times F_{\text {pred }}^{f}(z, T) 10^{-0.4 s^{f}} / \sigma_{\text {obs }}^{f}\right)$,

where $F_{\text {pred }}^{f}(z, T)$ is the predicted flux for a template $T$ at redshift $z, F_{\text {obs }}^{f}$ the observed flux, $\sigma_{\text {obs }}^{f}$ is the associated error, and $A$ is the normalization factor. The grid spacing in redshift is $\delta z=0.02$. A redshift probability distribution function $(\mathrm{PDFz})$ is computed for each object using the $\chi^{2}$ merit function, $\mathrm{PDFz}$ $\propto \exp \left(-\chi^{2}(z) / 2\right)$ every grid space. The best redshift is derived by parabolic interpolation of the PDF with galaxy library. If a second peak is found in the PDFz with a height larger than $5 \%$ of the main peak, the corresponding redshift is given as a second solution. In addition to the best $\chi^{2}$ derived from the galaxy library (hearafter $\chi_{\text {gal }}^{2}$ ), a best $\chi^{2}$ computed using the stellar liberally is derived for each object (hearafter $\chi_{\text {star }}^{2}$ ).

\subsection{Photometric redshift accuracy}

We assess the photometric redshift accuracy by comparing photometric redshifts against spectroscopic redshifts. Figure 10 shows the comparison between $z_{\mathrm{p}}$ with $z_{\mathrm{s}}$ from the 483 sources. Objects, whose second solution in the PDFz is found, are shown. Since $z_{\mathrm{p}}$ of those sources are not reliable, we excluded for the measurement of $z_{\mathrm{p}}$ accuracy. We obtained an accuracy of $\sigma_{\Delta z /(1+z)} \sim 0.032$ and a catastrophic error rate of $\eta=5.8 \%$ $\left(\frac{\Delta z}{1+z}>0.15\right)$ at $z<1$, while $\sigma_{\Delta z /(1+z)} \sim$ of 0.117 and $\eta=16.6 \%$ at $z>1$. The median offset between the photometric redshift and spectroscopic redshift is $\Delta z /(1+z)=0.008$ and 0.018 for $z<1$ and $z>1$, respectively.

Coupon et al. (2009) used the LePhare with the CFHTLS Deep and Wide T0004 catalogs $\left(u^{*}, g^{\prime}, r^{\prime}, i^{\prime}, z^{\prime}\right)$ to calculate $z_{\mathrm{p}}$ and measured the accuracy comparing with VVDS-deep and -wide, and DEEP2 (Davis et al. 2003, 2007) $z_{\mathrm{s}}$ samples. They find a dispersion of $0.028-0.030$ and an outlier rate of about

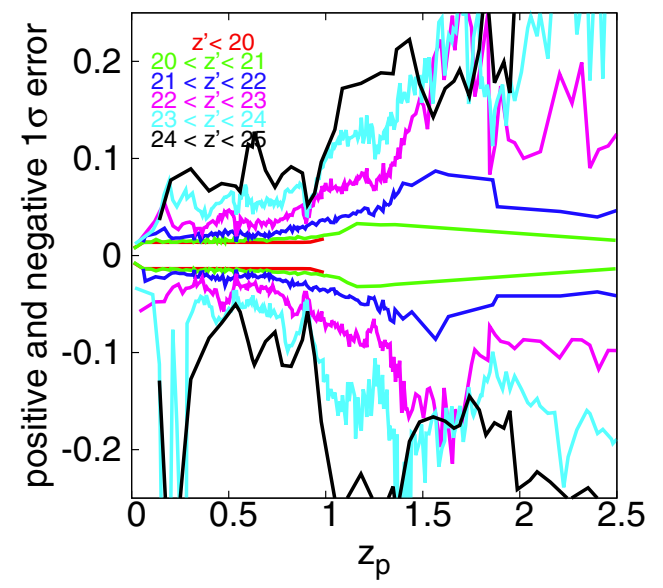

Fig. 11. $1 \sigma$ uncertainty for the $z_{\mathrm{p}}$ estimate as a function of photometric redshift in different $z^{\prime}$-band magnitude bins. Each value is computed with 100 galaxies per bin. The red, green, blue, pink, cyan, and black lines are $z^{\prime}<20,20<z^{\prime}<21,21<z^{\prime}<22,22<z^{\prime}<23,23<z^{\prime}<$ 24 , and $24<z^{\prime}<25$, respectively.

$3-4 \%$ in the range $17.5<i^{\prime}{ }_{\mathrm{AB}}<22.5$ at $0<z<2$. In our catalog, there are 382 sources with $z_{\mathrm{s}}$ in the range of $17.5<i_{\mathrm{AB}}^{\prime}<22.5$, and $97 \%$ of them are $z_{\mathrm{s}}<1$. We compared the $z_{\mathrm{p}}$ with their $z_{\mathrm{s}}$ and found an accuracy of $\sigma_{\Delta z /(1+z)} \sim 0.031$ with a catastrophic error rate of $\eta=3.5 \%$, which is comparable to the CFHTLS results.

Since evaluation of the $z_{\mathrm{p}}$ accuracy from the comparison with $z_{\mathrm{s}}$ is limited to a specific range of magnitude and redshift, we used the $1 \sigma$ uncertainty in the derived $z_{\mathrm{p}}$ probability distribution to extend the uncertainty estimates over the full magnitude/redshift space. For estimating the accuracy, we excluded stars. In Sect. 5, we securely flagged star using $\left(u^{*}-J\right)-\left(g^{\prime}-K_{\mathrm{s}}\right)$ color in addition to the stellarity index. During SED fitting using LePhare, we also calculated $\chi_{\text {gal }}^{2}$ and $\chi_{\text {star }}^{2}$ for each object. If $\chi_{\text {star }}^{2}<\chi_{\text {gal }}^{2}$, it suggests that the object is more likely to be a star than a galaxy. We compared the SED $\chi^{2}$ classification and color with the stellarity index, and found that both classifications agree with each other: 5381 out of 5441 star-flagged sources with the color-morphology classification method are classified as stars with the SED $\chi^{2}$ criterion. This suggests that both criteria work well, and then we excluded stars flagged by either SED $\chi^{2}$ classification or the color-morphology classification for measuring the $z_{\mathrm{p}}$ accuracy below.

Following Ilbert et al. (2006), we plotted the $1 \sigma$ negative and positive uncertainties derived from the PDFz as a function of redshift and $z^{\prime}$-band magnitude (Fig. 11). The uncertainties are derived from at $68 \%$ of the probability distribution. This figure shows that the accuracy is inevitably degraded for fainter galaxies at all redshifts, and the $z_{\mathrm{p}}$ have significantly higher uncertainty at $z_{\mathrm{p}} \gtrsim 1$. From $z_{\mathrm{p}}>0$ up to $z_{\mathrm{p}}=1$, the $1 \sigma$ errors do not dependent significantly on the redshift at $z^{\prime}<23$. With $z^{\prime}=23-$ 24 , the accuracy is $\sim 0.7$, which is consistent with the accuracy estimated from the $z_{\mathrm{p}}-z_{\mathrm{s}}$ comparison (see Fig. 10). For $z_{\mathrm{p}}>1$, the accuracy with $z^{\prime}<22$ is consistently low, while the accuracy for fainter sources worsen. Therefore, our photometric redshifts are highly accurate up to at least $z_{\mathrm{p}}<1$ with $z^{\prime}<24$ or $z_{\mathrm{p}}<2.5$ with $z^{\prime}<22$.

Figure 12 shows the photometric redshift distribution for all objects classified as galaxies with at least five band detections. The peak of the distribution is located at redshifts between 
N. Oi et al.: Optical and near-infrared merged catalog at AKARI NEP-Deep field

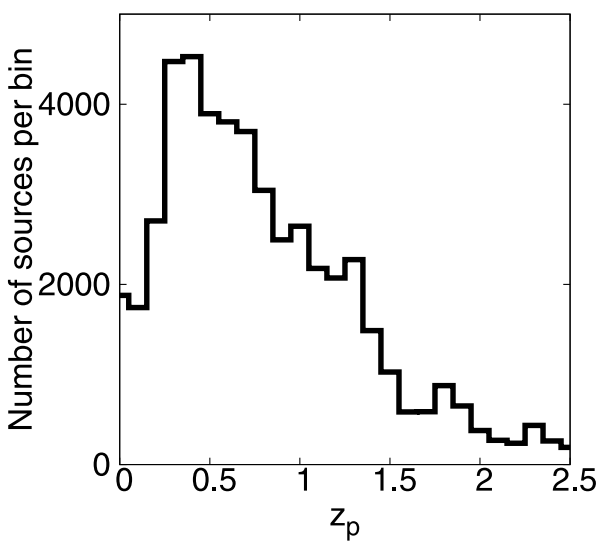

Fig. 12. Photometric redshift distribution between $0<z_{\mathrm{p}}<2.5$.

$z \sim 0.3-0.4$, and the number of sources monotonically decreases with increasing redshift.

\section{AKARI sources}

Murata et al. (2013) constructed a revised NIR to MIR catalog for the AKARI NEP-Deep survey. They compared the AKARI NEP-Deep catalog with our final catalog to identify optical counterparts. In total, 23345 AKARI sources have their optical counterparts, and 8916 of them have MIR (any of $S 7$ L24) fluxes. In this section, we focus on the sources detected by AKARI with optical counterparts and demonstrate the properties of the AKARI sources.

\section{1. $g^{\prime} z^{\prime} K_{s}$ diagram}

Daddi et al. (2004) developed a highly popular technique that allows both the selection and classification of $1.4<z<2.5$ starforming galaxies, passively evolved galaxies, and stars using a simple $B z K_{\mathrm{s}}$ color-color diagram. In the $B z K_{\mathrm{s}}$ technique, highredshift objects are uniquely located in regions of the $B z K_{\mathrm{s}}$ diagram. The validity of this technique has been supported by many authors (Hanami et al. 2012). The original paper of Daddi et al. (2004) used the VLT Bessel $B$ band filter, the VLT Gunn $z$ filter, and the ISAAC $K_{\mathrm{s}}$ band filter. Although the $z^{\prime}$ and $K_{\mathrm{s}}$-band filters of CFHT are similar to those that Daddi et al. used, the CFHT MegaCam $g^{\prime}$-band filter is offset from the $B$ band filter. ArcilaOsejo \& Sawicki (2013) considered the positions of the Daddi et al. (2004) models in the $g^{\prime} z^{\prime} K_{\mathrm{S}}$ color-color diagram to modify the $B z K_{\mathrm{s}}$ technique for use with MegaCam and WIRCam $g^{\prime} z^{\prime} K_{\mathrm{s}}$ filters. They find that even the $g^{\prime} z^{\prime} K_{\mathrm{s}}$ and $B z K_{\mathrm{S}}$ filter systems are different, the main qualitative features of the color-color diagram of models are similar enough. The $g^{\prime} z^{\prime} K_{\mathrm{S}}$ diagram can therefore be used for classifying $1.4<z<2.5$ star-forming galaxies, passively evolved galaxies, and stars. Arcila-Osejo \& Sawicki (2013) defined new color cuts to distinguish those properties as

$\left(z^{\prime}-K_{\mathrm{S}}\right)-1.27\left(g^{\prime}-z^{\prime}\right)=-0.022$

$\left(z^{\prime}-K_{\mathrm{S}}\right)-1.27\left(g^{\prime}-z^{\prime}\right)=-0.022 \cap\left(z^{\prime}-K_{\mathrm{S}}\right)=2.55$

$\left(z^{\prime}-K_{\mathrm{S}}\right)-0.45\left(g^{\prime}-z^{\prime}\right)=-0.57$.

As the same as $B z K_{\mathrm{s}}$ technique, high-redshift star-forming galaxies are located on the lefthand side of Eq. (2), and stars are separated by Eq. (4) in the $g^{\prime} z^{\prime} K_{\mathrm{S}}$ diagram.

We first plotted the $z_{\mathrm{p}}<1.4$ AKARI sources (left panel of Fig. 13). In the figure, almost all stars ( 98.9\%) classified
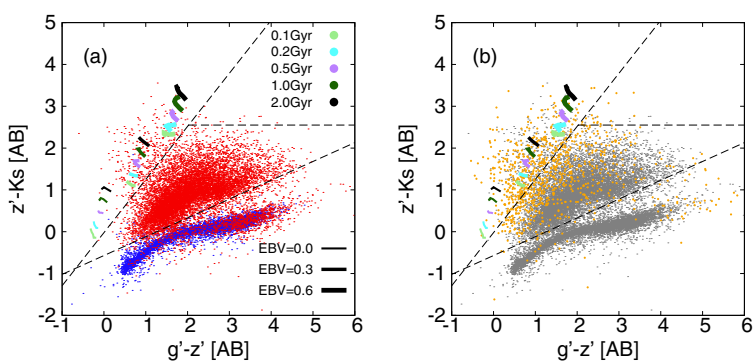

Fig. 13. $g^{\prime} z^{\prime} K_{\mathrm{s}}$ diagram for AKARI sources with optical counterparts. (Left) blue dots represent stars identified in Sect. 5, and red dots indicate galaxies with $z_{\mathrm{p}}<1.4$. The dashed lines are the borders given by Eqs. (2)-(4). Bruzual \& Charlot (2003) stellar population synthesis models for $g^{\prime} z^{\prime} K_{\mathrm{s}}$ color-color diagram with $1.4<z_{\mathrm{p}}<2.5$, which Arcila-Osejo \& Sawicki (2013) show are overplotted for comparison. Ages in Gyr are shown with different colors, while different $E(B-V)$ are represented with different line thicknesses. (Right) $1.4<z_{\mathrm{p}}<2.5$ AKARI sources are plotted in yellow. The $z_{\mathrm{p}}<1.4$ sources shown in the left panel are also plotted but in gray.

in Sect. 5 are found under Eq. (4), while extended galaxies are located in the area enclosed by the three equations. It suggests that the criteria of star-galaxy classification discussed in Sect. 5 work well. On the other hand, we plotted galaxies at $1.4<z_{\mathrm{p}}<2.5$ in the $g^{\prime} z^{\prime} K_{\mathrm{s}}$ diagram in the righthand panel of Fig. 13. All points in the lefthand panel are plotted. We found that the $1.4<z_{\mathrm{p}}<2.5$ galaxies are systematically located in the upper left of galaxies of $z_{\mathrm{p}}<1.4$, although more than $50 \%$ of $1.4<z_{\mathrm{p}}<2.5$ galaxies are scattered into the $z_{\mathrm{p}}<1.4$ region. This is probably a consequence of the increased scatter of the photometric redshift that is higher than $z_{\mathrm{p}}>1$. When we use the $g^{\prime} z^{\prime} K_{\text {s }}$ diagram, we can distinguish 759 AKARI sources $(\sim 3.2 \%)$ as high- $z$ galaxies independent of $z_{\mathrm{p}}$ with large uncertainty at $z_{\mathrm{p}}>1$. The average $z^{\prime}$ magnitude of the high- $z$ galaxies of $1.4<z_{\mathrm{p}}$ is $23.2( \pm 0.4) \mathrm{mag}$, which is consistent with the $z^{\prime}$-band $50 \%$ completeness, suggesting that it is possible there are AKARI sources of high- $z$ star-forming galaxies without optical counterparts. We also found that only less than $0.1 \%$ of AKARI sources come into the passive galaxies' area above Eq. (3).

Seen from the $g^{\prime} z^{\prime} K_{\mathrm{s}}$ diagram, the AKARI sample is dominated by sources at $z<1.4$, and $<5 \%$ of the AKARI sources with optical counterparts are located in the high- $z$ universe, and most of them seem to be star-forming active galaxies.

\subsection{Mid-infrared sources}

We compared $z_{\mathrm{p}}$ between sources with detections and nondetections in AKARI MIR bands. We divided AKARI sources into two groups: one is detected in one of the MIR bands ( $S 7-$ L24) and the other is not. After excluding star-flagged sources, the MIR detected and non-detected groups include 8085 sources and 11616 sources, respectively. Figure 14 shows $z_{\mathrm{p}}$ histograms for each group. This figure shows that the non-detected MIR group is systematically distributed at a higher redshift than the MIR detected group. The distribution of the MIR-detected group has a sharp peak around $z_{\mathrm{p}} \sim 0.5$ and suddenly decreases at higher redshift, while MIR non-detected sources have gentle peak around $z_{\mathrm{p}} \sim 0.5-1$. These tendencies are explained by the difference in sensitivities of the AKARI NIR and MIR detectors. Since MIR bands' sensitivities are relatively poorer than those of NIR bands, there is a bias toward MIR bands detecting lower redshift bright sources. 


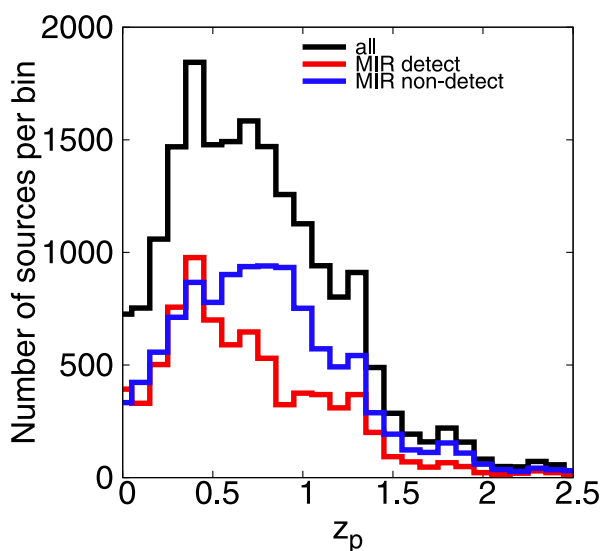

Fig. 14. Histograms of $z_{\mathrm{p}}$ for AKARI sources at $0<z_{\mathrm{p}}<2.5$. Sources flagged as stars in Sect. 5 are excluded. Black, red, and blue lines represent all sources, sources with and without MIR detections, respectively.

Finally we plotted the AKARI sources in the $g^{\prime} z^{\prime} K_{\mathrm{s}}$ plane in Fig. 15. This figure shows that some sources with non-detections in AKARI MIR bands $(\sim 22 \%)$ are plotted in the stellar region under Eq. (4), while stellar contamination of the sources with detections in the MIR bands are negligible small ( 4.0\%). A very similar fraction of sources with and without MIR detections $(\sim 5.3 \%$ and $\sim 6.4 \%$, respectively) are distributed in the $1.4<z<$ 2.5 star-forming galaxy region.

For those sources located in the $1.4<z<2.5$ star-forming galaxy region, MIR-detected sources seem to be systematically redder along the line of Eq. (2) than for the MIR non-detected group. Since galaxies become redder along the line of Eq. (2) depending on the $E(B-V)$ values in the Bruzual \& Charlot (2003) model as shown in Fig. 13, here we have defined an axis along the line of Eq. (2) as the "reddening axis". The difference between MIR-detected and non-detected groups can be more clearly seen along the "reddening axis" as shown in the righthand panel of Fig. 15. Although the color can be redder both from the old age and the star formation along the "reddening axis", the effect from the age is significantly small. Therefore, the redder color of MIR-detected sources probably indicates that they are dust-attenuated star-forming galaxies.

In conclusion, when we select sources detected in AKARI MIR bands, then dusty, star-forming galaxies at around $z_{\mathrm{p}} \lesssim 0.5$ tend to be chosen with less stellar contamination.

\section{Summary and conclusion}

We obtained $g^{\prime}, r^{\prime}, i^{\prime}, z^{\prime}, Y, J$, and $K_{\mathrm{s}}$-band images with MegaCam and WIRCam at CFHT, and cross-matched each of all the band catalogs with the existing $u^{*}$-band catalog to construct an optical to NIR eight-band, merged photometric catalog covering the AKARI NEP-Deep field. The $4 \sigma$ detection limits over a 1 arcsec radius aperture are 26.7, 25.9, 25.1, 24.1, 23.4, 23.0, and 22.7 $A B$ mag for $g^{\prime}, r^{\prime}, i^{\prime}, z^{\prime}, Y, J$, and $K_{\mathrm{s}}$-bands, respectively. The astrometry of the band-merged catalog is offset by 0.013 arcsec to the 2MASS with an RMS offset of 0.32 arcsec, while the RMS offsets between $z^{\prime}$-band and the other bands are $0.08-0.11$ arcsec, but between $z^{\prime}$ and $u^{*}$-bands it is worse $\left(0.18\right.$ arcsec) because of a slightly degraded PSF in the $u^{*}$-band. We securely distinguished stars from galaxies with a $\left(u^{*}-J\right)$ versus $\left(g^{\prime}-K_{\mathrm{s}}\right)$ color-color diagram with CLASS_STAR of $<0.8$. We derived photometric redshifts based on an SED template fitting procedure using LePhare. The errors comparison with $z_{\mathrm{s}}$ for our spectroscopic subsample reveals the dispersion
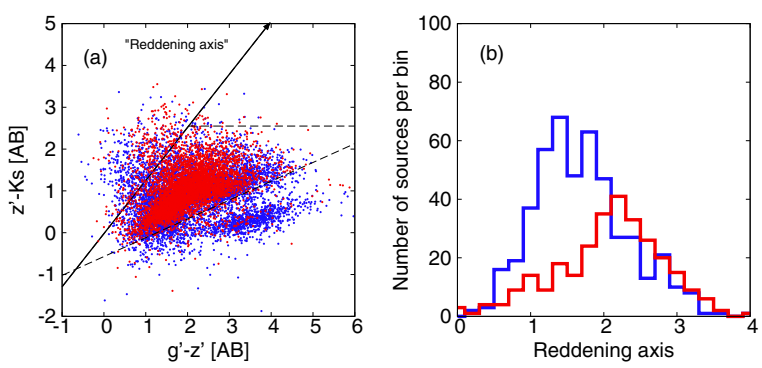

Fig. 15. Left: $g^{\prime} z^{\prime} K_{\mathrm{s}}$ diagram for sources with and without detections in AKARI MIR bands. The color of indices are the same as in Fig. 14. The solid thick arrow is the axis defined along the line of Eq. (2) named "reddening axis" (see text). Right: distribution along the "reddening axis" for AKARI high- $z$ star-forming sources above Eq. (2) in the left panel. The values along the "reddening axis" are calculated by coordinate transformation from the $g^{\prime} z^{\prime} K_{\mathrm{s}}$ diagram.

of $\sigma_{\Delta z /(1+z)} \sim 0.032$ with $\eta=5.8 \%$ catastrophic failures at $z_{\mathrm{p}}<1$. We extrapolate this result to fainter magnitudes using the $1 \sigma$ uncertainties in the $z_{\mathrm{p}}$ probability distribution functions. At $z_{\mathrm{p}}<1$, we estimate a $z_{\mathrm{p}}$ accuracy of $\sigma_{\Delta z}=0.7$ with $z^{\prime}<24$, while the accuracy is strongly degraded at $z_{\mathrm{p}}>1$ or $z^{\prime}>24$. We investigated properties of AKARI sources with optical counterparts from our catalog. We plotted the sources with the $g^{\prime} z^{\prime} K_{\mathrm{S}}$ diagram and found that most of the AKARI sources are located in the low $-z(z<1.4)$, and $<5 \%$ of them are classified as high- $z(1.4<z<2.5)$ star-forming galaxies. Among the high- $z$ star-forming galaxies, MIR detected sources seem to be affected by stronger dust extinction compared with sources with non-detections in AKARI MIR bands. Our final catalog contains 85797 sources with $z_{\mathrm{p}}$, which are detected in the $z^{\prime}$ band and in at least one of the other bands. This optical to NIR photometric data with photometric redshift in the AKARI NEP-Deep field derived here are crucial to studying star formation history at the era of active universe $(z \sim 1)$ using multiwavelength data sets (Chandra, GALEX, AKARI, Herschel, SCUBA-2, and WSRT).

Acknowledgements. This work is supported by the Japan Society for the Promotion of Science (JSPS; grant number 23244040) and grant NSC 100-2112M-001-001-MY3 (Y.O.). M.I. acknowledges the support from a grant No. 20080060644 of CRI/NRFK/MSIP of Korea. This work is based on observations obtained with MegaPrime/MegaCam, a joint project of CFHT and CEA/DAPNIA, and WIRCam, a joint project of CFHT, Taiwan, Korea, Canada, France, at the Canada-France-Hawaii Telescope (CFHT), which is operated by the National Research Council (NRC) of Canada, the Institute National des Sciences de l'Univers of the Centre National de la Recherche Scientifique of France, and the University of Hawaii. S. Foucaud (National Taiwan Normal University) is acknowledged for this modified SWarp in Sect. 2.

\section{Appendix A: Catalog format}

We give a short description of the columns in the catalog in the following.

Column (1): identification number.

Columns (2) and (3): the $J 2000$ right ascension and the declination, in degrees. The coordinates are based on the $z^{\prime}$-band astrometry.

Columns (4) and (5): the AB magnitudes of the sources in the $z^{\prime}$-band, and $z^{\prime}$-band magnitude error, respectively.

Columns (6), (9), (12), (15), (18), (21), and (24): the AB magnitudes of the other bands $\left(u^{*}, g^{\prime}, r^{\prime}, i^{\prime}, Y, J\right.$, and $K_{\mathrm{s}}$-bands).

Columns (7), (10), (13), (16), (19), (22), and (25): the magnitude errors for the other bands.

Columns (8), (11), (14), (17), (20), (23), and (26): the crossmatching flags. " 0 ": a unique counterpart is found for the $z^{\prime}$-band 
sources. "1": multiple counterparts are found for the $z^{\prime}$-band sources. The magnitude is the sum of all the candidate fluxes. "2": multiple $z$-band sources have one common counterpart in the other band. If counterparts are not found, "-99.00" is inserted as the dummy values into magnitude and error columns of the no-counterpart bands with a " -1 " flag.

Column (27): the CLASS_STAR at $z^{\prime}$-band image.

Column (28): flags of stars based on color-color diagram. "STAR" is identified as a star and "****" the others.

Columns (29) and (30): spectroscopic redshift and flag measured by Takagi et al. (in prep.) using Keck/DEIMOS. "5": secure redshift with multiple lines, including spectroscopically resolved [OII] doublet. "3": redshift from absorption lines only. "2": redshift from single line, probably [OII]. "1": single line with less confidence. "-1": problem in data. "-3": redshift from absorption lines alone, with problems in data. "-5": secure redshift, but problems in data.

Columns (31), (32), and (33): spectroscopic redshift, flag, and classification by Shim et al. (2013), using MMT/Hectospec and WIYN/HYDRA. "4.0": the redshift is clearly identified using more than two significant spectral features. "3.0": the redshift is very probably identified. Multiple spectral features are used, but some of the features have a low signal-to-noise ratio. "2.5": objects which seem to be between flags 2 and 3. "2.0": the redshift is identified by single, weak spectral features (including continuum break). Probable, but unreliable. "1.5": the spectral features are very weak, might be real, but are not reliable. There are five classifications; GALAXY, STAR, TYPE1, TYPE2, and UNKNOWN.

Column (34): photometric redshift for galaxy measured by SED fitting (Sect. 6). Star-flagged sources in Col. (28) have a value of 0.000 .

Column(35): Classes (star/galaxy) from SED fitting $\chi^{2}$ values.

\section{References}

Arcila-Osejo, L., \& Sawicki, M. 2013, MNRAS, 435, 845

Arnouts, S., Walcher, C. J., Le Fèvre, O., et al. 2007, A\&A, 476, 137

Beers, T. C., Flynn, K., \& Gebhardt, K. 1990, AJ, 100, 32

Bertin, E. 2006, in Astronomical Data Analysis Software and Systems XV, eds

C. Gabriel, C. Arviset, D. Ponz, \& S. Enrique, ASP Conf. Ser., 351, 112

Bertin, E., \& Arnouts, S. 1996, A\&AS, 117, 393

Bertin, E., Mellier, Y., Radovich, M., et al. 2002, in Astronomical Data Analysis Software and Systems XI, eds. D. A. Bohlender, D. Durand, \& T. H. Handley, ASP Conf. Ser., 281, 228

Bielby, R., Hudelot, P., McCracken, H. J., et al. 2012, A\&A, 545, A23

Bohlin, R. C., Colina, L., \& Finley, D. S. 1995, AJ, 110, 1316

Boulade, O., Charlot, X., Abbon, P., et al. 2003, in SPIE Conf. Ser. 4841, eds. M. Iye, \& A. F. M. Moorwood, 72

Brodwin, M., Brown, M. J. I., Ashby, M. L. N., et al. 2006, ApJ, 651, 791 Bruzual, G., \& Charlot, S. 2003, MNRAS, 344, 1000

Buat, V., Takeuchi, T. T., Iglesias-Páramo, J., et al. 2007, ApJS, 173, 404

Calzetti, D., Armus, L., Bohlin, R. C., et al. 2000, ApJ, 533, 682
Choi, P. I., Yan, L., Im, M., et al. 2006, ApJ, 637, 227

Coleman, G. D., Wu, C.-C., \& Weedman, D. W. 1980, ApJS, 43, 393

Connolly, A. J., Szalay, A. S., Dickinson, M., Subbarao, M. U., \& Brunner, R. J. 1997, ApJ, 486, L11

Coupon, J., Ilbert, O., Kilbinger, M., et al. 2009, A\&A, 500, 981

Daddi, E., Cimatti, A., Renzini, A., et al. 2004, ApJ, 617, 746

Davis, M., Faber, S. M., Newman, J., et al. 2003, in Discoveries and Research Prospects from 6- to 10-Meter-Class Telescopes II, ed. P. Guhathakurta, SPIE Conf. Ser., 4834, 161

Davis, M., Guhathakurta, P., Konidaris, N. P., et al. 2007, ApJ, 660, L1

Fazio, G. G., Hora, J. L., Allen, L. E., et al. 2004, ApJS, 154, 10

Finlator, K., Ivezić, Ž., Fan, X., et al. 2000, AJ, 120, 2615

Franceschini, A., Rodighiero, G., \& Vaccari, M. 2008, A\&A, 487, 837

Girardi, L., Barbieri, M., Groenewegen, M. A. T., et al. 2012, TRILEGAL, a

TRIdimensional modeL of thE GALaxy: Status and Future, eds. A. Miglio, J. Montalbán, \& A. Noels, 165

Goto, T., Takagi, T., Matsuhara, H., et al. 2010, A\&A, 514, A6

Hanami, H., Ishigaki, T., Fujishiro, N., et al. 2012, PASJ, 64, 70

Hicks, E. K. S., Malkan, M. A., Teplitz, H. I., McCarthy, P. J., \& Yan, L. 2002, ApJ, 581, 205

Hopkins, A. M., Connolly, A. J., Haarsma, D. B., \& Cram, L. E. 2001, AJ, 122, 288

Houck, J. R., Roellig, T. L., van Cleve, J., et al. 2004, ApJS, 154, 18

Hwang, N., Lee, M. G., Lee, H. M., et al. 2007, ApJS, 172, 583

Ilbert, O., Arnouts, S., McCracken, H. J., et al. 2006, A\&A, 457, 841

Ilbert, O., Capak, P., Salvato, M., et al. 2009, ApJ, 690, 1236

Imai, K., Matsuhara, H., Oyabu, S., et al. 2007, AJ, 133, 2418

Kessler, M. F., Steinz, J. A., Anderegg, M. E., et al. 1996, A\&A, 315, L27

Kinney, A. L., Calzetti, D., Bohlin, R. C., et al. 1996, ApJ, 467, 38

Kron, R. G. 1980, ApJS, 43, 305

Lagache, G., Abergel, A., Boulanger, F., Désert, F. X., \& Puget, J.-L. 1999, A\&A, 344, 322

Ly, C., Malkan, M. A., Kashikawa, N., et al. 2007, ApJ, 657, 738

Madau, P. 1995, ApJ, 441, 18

Magnier, E. A., \& Cuillandre, J.-C. 2004, PASP, 116, 449

Marmo, C., \& Bertin, E. 2008, in Astronomical Data Analysis Software and Systems XVII, eds. R. W. Argyle, P. S. Bunclark, \& J. R. Lewis, ASP Conf. Ser., 394, 619

Massarotti, M., Iovino, A., Buzzoni, A., \& Valls-Gabaud, D. 2001, A\&A, 380, 425

Matsuhara, H., Wada, T., Matsuura, S., et al. 2006, PASJ, 58, 673

Murakami, H., Baba, H., Barthel, P., et al. 2007, PASJ, 59, 369

Murata, K., Matsuhara, H., Wada, T., et al. 2013, A\&A, 559, A132

Neugebauer, G., Habing, H. J., van Duinen, R., et al. 1984, ApJ, 278, L1

Onaka, T., Matsuhara, H., Wada, T., et al. 2007, PASJ, 59, 401

Pickles, A. J. 1998, PASP, 110, 863

Prevot, M. L., Lequeux, J., Prevot, L., Maurice, E., \& Rocca-Volmerange, B. 1984, A\&A, 132, 389

Puget, J.-L., Abergel, A., Bernard, J.-P., et al. 1996, A\&A, 308, L5

Puget, P., Stadler, E., Doyon, R., et al. 2004, in SPIE Conf. Ser. 5492, eds. A. F. M. Moorwood, \& M. Iye, 978

Rieke, G. H., Young, E. T., Engelbracht, C. W., et al. 2004, ApJS, 154, 25

Shim, H., Im, M., Ko, J., et al. 2013, ApJS, 207, 37

Stecker, F. W., Malkan, M. A., \& Scully, S. T. 2006, ApJ, 648, 774

Steidel, C. C., Adelberger, K. L., Giavalisco, M., Dickinson, M., \& Pettini, M. 1999, ApJ, 519, 1

Strateva, I., Ivezić, Ž., Knapp, G. R., et al. 2001, AJ, 122, 1861

Sullivan, M., Mobasher, B., Chan, B., et al. 2001, ApJ, 558, 72

Takagi, T., Matsuhara, H., Goto, T., et al. 2012, A\&A, 537, A24

Wada, T., Matsuhara, H., Oyabu, S., et al. 2008, PASJ, 60, 517

York, D. G., Adelman, J., Anderson, Jr., J. E., et al. 2000, AJ, 120, 1579 